\title{
Thermodynamics and kinetics of non-autonomous phase formation in nanostructured materials with variable functional properties
}

\author{
A. N. Kovalenko ${ }^{1}$, E. A. Tugova ${ }^{1,2}$ \\ ${ }^{1}$ Ioffe Institute, 26 Politekhnicheskaya, St. Petersburg 194021, Russia \\ ${ }^{2}$ St. Petersburg Electrotechnical University "LETI", ul. Professora Popova 5, St. Petersburg 197376, Russia \\ ras-kan@mail.ru, katugova@inbox.ru
}

PACS 05.70.Ce; 05.70.Ln; 64.60.Qb

DOI 10.17586/2220-8054-2018-9-5-641-662

\begin{abstract}
The review addresses physico-chemical aspects of interaction between macro-, micro- and nano- structured units of matter with the analysis of interface and grain boundary entities (nonautonomous phases) mechanisms and formation, as well as methods of their control in order to achieve the desired functional properties of nanostructured materials. Construction of these materials involves identification of thermodynamic and kinetic regularities in the organization processes, state and genesis parameters of nonautonomous phases formed as specific nanosized structures in a limited space between the macroscopic volume phases and with the limited amount of substance, which differ significantly on their properties, structure and composition from the appropriate characteristics of volume phases. Studying them is based on the application and development of theoretical and experimental methods of non-equilibrium thermodynamics, chemical kinetics, nonlinear dynamics and fractal analysis to determine the conditions of self-organization or materials directed synthesis with a high content of nonautonomous phases.
\end{abstract}

Keywords: nanostructered materials, non-equilibrium thermodynamic processes, chemical kinetics, interaction of macro-, micro- and nanostructured elements, nonautonomous phases, conditions of self-organization, materials synthesis, functional properties.

Received: 1 May 2018

Revised: 30 July 2018

\section{Contents}

1. Introduction

2. Status of nonautonomous phases and materials with the high value of matter in the nonautonomous phases study

3. Thermodynamics of the systems which include nonautonomous interphase areas and conjugated macroscale substance volumes

4. Heat-and-mass transfer and kinetics of chemical reactions in materials with a high content of nonautonomous phases

5. Conclusion

\section{Introduction}

The nonautonomous phase concept regards small objects formed at the junctions between the macroscale substance volumes as intermediate (transient) surface, interface or grain boundary structures which composition, structure and properties are determined by spatial or substance amount limitations, and which cannot exist as independent thermodynamic phases. Nonautonomous phases are highly important, and previously, a rarely considered part of synthesized materials, whereas determining their functional properties in many respects. Development of this concept as a physical and chemical basis for designing new functional materials, including nanocomposites, involves focusing on interactions between their macro-, micro- and nanostructured units using various methods and approaches, including theoretical and experimental methods of non-equilibrium thermodynamics, chemical kinetics, nonlinear dynamics and fractal analysis.

It would be feasible to apply these methods in addressing the given problem due to the ability to describe both equilibrium and non-equilibrium systems, with due account of metastable and intermediate structures formed as a result of these interactions. Herewith, it is of great interest to reveal the regularities of formation and state parameters of rather stable nonautonomous phases, the causes of which may be thermodynamic and kinetic characteristics of interactions between different-scaled substance structures. It is essential to identify the conditions of self-organization, as well as methods to deliberately form the nonautonomous phases with distinct physical and chemical, mechanical, thermal, electrical, magnetic and other properties, as compared to volume phases [1-25]. Nonautonomous phases are also able to concentrate impurity elements in ultrahigh concentrations [26-29]. The latter property results in significant redistribution of components between the surface (interfacial, intergranular) 
formations, i.e. non-autonomous phases, and the volume phases in the materials [30-35]. Such phenomena related to the formation of nonautonomous phases as material surface nanofragmentation invoking the nanocrystalline structure fractality, improved adsorption and catalytic properties, are also of great interest [36-48].

The study of these effects by methods of non-equilibrium thermodynamics, nonlinear dynamics and chemical kinetics is aimed at identifying mechanisms and energy of the occurring processes and their dynamic parameters, in particular, in the phase space. Probabilistic multifractal dimensions can be used to quantify the scale invariance of these structures, manifested in this case, and the attractor of entropy generated in them during the substance and energy transfer. Their general expression is the Renyi dimension $[49,50]$ which describes metric and statistical properties of these materials and processes occurring in them.

Experimental studies of structural and chemical transformations in the systems containing a large substance proportion in the nonautonomous phases, as well as of physical-chemical, mechanical, thermal physical, electrophysical, magnetic and other properties of materials based on these systems are of independent interest. Since these materials contain nanoscale elements, not only their properties but also their geometric characteristics, as well as the geometric parameters of their fragments, can be determined only based on the analysis of a set of complementary methods [51-54]. The synthesis of such materials requires the development of special approaches and methods, and represents a standalone target [55-65].

This review does not address a number of the above issues related to the development of materials with a high substance proportion in the nonautonomous phases, and is aimed primarily at describing of nonautonomous phases and materials based on systems in which a large substance proportion resides in nonautonomous phases behavior.

\section{Status of nonautonomous phases and materials with the high value of matter in the nonautonomous phases study}

Nonautonomous phases, as specific small-scale structures existing in a confined space between the macroscopic volume phases, or in a limited substance amount located on a surface, are significantly different in their properties, behavior and structure from the volume phases while similar in terms of chemical composition [66-73]. They are characterized by different melting temperatures, other phase transitions [74-85], and other critical parameters. Materials containing a substantial portion of nonautonomous phases show peculiar deformation characteristics, different behavior when the temperature varies due to changes in thermodynamic and transport properties of such materials [86-91]. The fundamental and applied research being done in this field is largely focused on the study of structural and functional characteristics of such materials, and on the development of technological methods to produce nano-dispersed, nano-crystalline and nano-porous materials [92-101]. Less attention is paid to research of physical and chemical mechanisms and conditions of formation and transformation of nonautonomous phases with a specific composition and structure, including the relationship between thermodynamic and other characteristics of similar nanoscale formations and their topological and metric structure, processes of environmental interaction, the extent of influence on the properties of spatially conjugated macroscopic volume phases.

Research in the field of objects, which are formed at the intersection between substance macro-volumes as intermediate (surface, interfacial) structures, dates back to the papers by T. Young (1805-1807), J. W. Gibbs (1878) and J.D. Van der Waals (1908-1912). In [102], the surface phases (thermodynamic substance state at the interface of volume phases) are described as "finite-thickness phases", and in [103,104], these finite-thickness phases were discussed in the context of equilibrium thermodynamics as "nonautonomous phases", meaning that their independent (isolated from the volume phases) existence is impossible. In [1,105-107], the concept of "nonautonomous phases" was transferred to a wider range of objects and expanded to the concept of "nonautonomous substance state", i.e. defining such a state of substance in which the composition, structure and properties are determined by various spatial limitations, i.e., depend fundamentally on the size and shape of the limiting surfaces, on the substance amount within a limited space, on the composition and structure of the limiting walls [1].

Thermodynamic ideas about the substance behavior on the interface of volume phases, and about the role of phase interfaces in the system behavior are developing in two directions to date. Historically, the first idea of the substance properties in the phase interface is based on their reference to the phase interfacial area $[108,109]$. In this case, we lose information about the structure and distribution of the substance over the transition area thickness (over the nonautonomous finite-thickness phase formed at the interface between the two phases. It should be noted that this approach, as shown in $[75,82,87]$, is not very effective in the analysis of kinetic processes as well, and in cases where it is required to compare the substance volume fractions in the nonautonomous and volume phases, i.e., primarily in nanoscale systems. The second approach is based on addressing the nonautonomous phases as those having a certain finite thickness, the substance of which has a specific structure. It was shown in a number of papers (for instance, [110-112]) that both approaches are equivalent in the context of equilibrium thermodynamics, which is a certain theoretical justification of equal, in a way, existence of both approaches. 
Description of the behaviors of polycrystalline materials, other disperse systems, especially those consisting of nanoscale areas of autonomous phases, as well as of the materials at high temperatures, as shown in numerous papers [75, 78-82], gives more prognostic conclusions if the interface areas formed by contact of volume phases are considered as nonautonomous finite-thickness phases. In this approach, determining such parameters as the thickness of nonautonomous phases and their melting point is of considerable interest in the analysis of the behavior of both single- and multiphase powder and polycrystalline materials. $[1,75,82,87,113-116]$ lists a variety of expressions to describe the temperature dependence of the nonautonomous phase thickness. The basic conclusion from these expressions is the fact that if the nonautonomous phase thickness has nanometer and sub-nanometer dimensions at relatively low temperatures, it increases sharply with temperature rise and with the approach to the melting point for solids, or to the critical point for liquids, thus becoming comparable to the size of volume phases. The most detailed variant of this dependence seems to be achieved in [117]. Another important property of the nonautonomous finite-thickness phase is its melting point. If early papers $[75,78,80,81,117,118]$ provided discussion that the melting temperature of surface (nonautonomous) phases may differ from the melting temperature of volume phases of the same composition, the recent papers generally consider only the value of these differences and expressions describing the dependence of the melting temperature of different nonautonomous phases on certain parameters $[13,15,95,97-99,108,214]$. Information on the component distribution between the nonautonomous and volume phases, which makes it possible to predict the material properties and behavior over a wide temperature range, is of great importance for the analysis of polycrystalline and other disperse systems $[78,79,117]$. In this respect, it should be noted that there is a significant advantage, explained in detail in [78,82], of considering the component redistribution between the volume phases and the nonautonomous finite-thickness phases, as compared to the approach considering the concentration of impurity components at the volume phase interface as their adsorption or segregation on a two-dimensional surface. However, the latter way of consideration is widespread and also provides useful information for analyzing the properties and behavior of materials $[1,6,13,15,71,95,97-$ 99, 105-107].

Consideration of nonautonomous phases as finite-thickness ones enables to analyze, in particular, the possibility of forming in them not only static but also dynamic structures resulting out of transformation processes and mass transfer in them $[84,119-125]$. This possibility was used to analyze the behavior of both synthetic materials [1, $6,13,15,71,95,97-99,105-107,112,123]$ and natural objects, in particular, in the behavioral analysis of ice and massive bodies in ice [113,126-130].

The structure and contribution of nonautonomous phases and properties of polycrystalline materials are greatly influenced by the size and misalignment degree of crystallites in their composition [131-135]. The thickness, structure, composition, and properties of the intercrystalline layer (nonautonomous phase) vary by the crystallite misalignment degree $[1,13,27,30,40,59,71,73,85]$. The ratio between crystallite size and intercrystalline layer thickness (nonautonomous surface phase), in turn, determines the volume fractions of crystallites and intercrystalline formations in the polycrystalline material $[75,78-82,105,106]$. Moreover, any chemical interaction during a solidphase reaction involves the mass transfer of components across the phase interface. For solid-phase reactions, the reaction zone is localized at the phase interface which is structurally heterogeneous, and therefore heterogeneous in its chemical activity. According to the data available [75,78-82], the mass transfer of components is much swifter in nonautonomous phases (surface, grain boundary, interface areas) than in granule volume under certain conditions. The interest in considering nonautonomous phases as objects with properties unlike those of volume phases is more related to their melting processes. The process of melting is essential for describing the solid-phase process activation which occurs at the melting point of the nonautonomous phase $[1,13,15,59,62,75-82,136,137]$. Melting of nonautonomous phases in polycrystalline systems is a key transformation that generally affects the system behavior, while the melting point of nonautonomous phase $\left(T_{m 2 n}\right)$ becomes one of the key parameters relating to the outset of transformation $[1,13,15,59,62,75-82,136,137]$. As shown in [75-82], the mass transfer of a substance will be carried out mainly in liquid-like nonautonomous phases in dispersed, including heterophase, systems, especially in case of small, nanoscale and submicron, granular sizes at temperatures $T>T_{m 2 n}$.

This statement is supported, for instance, by the case of autocatalytic solid-phase reaction of chrysoberyl formation, as described in [138]. A large number of papers, both on the subject of analysis of the activation temperature in sintering and inelastic deformation of materials $[139,140]$ and on that of the analysis of solidphase reaction mechanisms [141-151], show that all these solid-phase processes start in the systems only after the nonautonomous phase has transitioned to a liquid-like state, i.e., they are liquid-phase reactions in a sense, as stated in $[75,78]$. As another example, we can compare the findings from the study of Ruddlesden-Popper phase formation kinetics from different reagents [151-168] with data on the values of some temperature transformations in the $\mathrm{Ln}_{2} \mathrm{O}_{3}-\mathrm{SrO}-\mathrm{Al}_{2} \mathrm{O}_{3}$ system [169-173], in which the compounds are formed that are promising for the production of functional and structural high-temperature materials [174-180]. This comparison has shown that 
solid-phase reactions are activated in the system considered only after the nonautonomous phases have transited to a liquid-like state, as may be concluded on the basis of these papers $[1,15,59,75,78-82,84,133,138-140,142,144]$.

The transition to nano-sized reagent particles leads to a significant increase in the volume fraction of nonautonomous phases in the reaction system $[1,117,118]$. The diagram shown in Fig. 1 illustrates the relationship between nonautonomous phase fraction and the particle size.

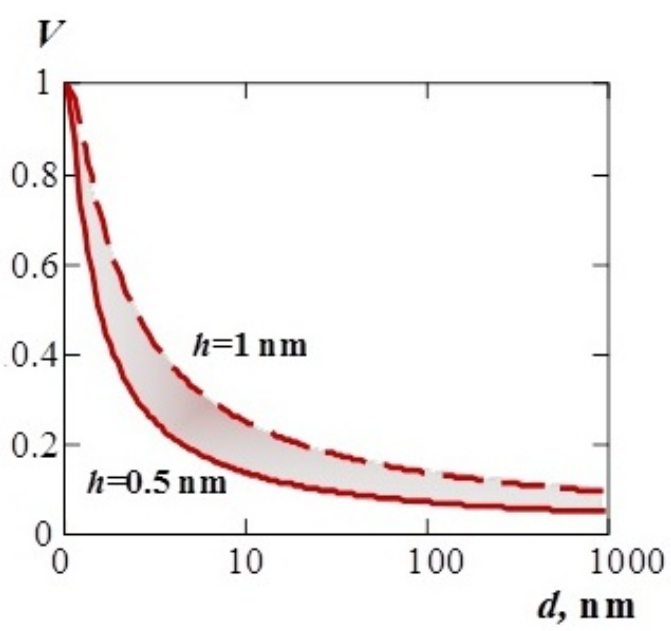

FIG. 1. Dependence of nonautonomous phase volume fraction in disperse system $v s$ autonomous phase particle sizes [1]

A sharp increase in nonautonomous phase fraction in the area of nanoscale particles shows that the use of nanoscale reagents should intensify the interaction between the solid-phase components. Chemical reactions between nanoparticles should go much faster than in the case of the interaction between micro- and macroscale particles. In this regard, it seems important to develop methods which produce nanocrystalline powders of various compositions that could be precursors for the synthesis of multicomponent compounds [181-191]. However, as shown in [12,60,145], reducing the size of reagent particles would not imply a certain increase in the speed of their interaction. At the same time, a temperature rise above the melting point of nonautonomous phases, as shown by numerous literature data, always results in a sharp increase in the speed of component interaction in solid-phase systems. In view of the above findings, it can be concluded that an important line of research is the study of phase formation mechanisms in the systems with nanoscale reagents, inter alia, with nanoscale spatial limitations, i.e. of the processes in nanoreactors [17,192-195]. Not only the "soft chemistry" methods [5,13,37,41,59,97,196,197], but also the methods based on precursor combustion [11,61,95,99,190,198-200], in particular, the solvent combustion method [11, 16, 21, 22, 62, 95,99, 190, 198-200] are effective for producing materials with a high proportion of nonautonomous phases.

The features of nanodispersed systems related to the phenomenon of "quantum-dimensional effects", to the changes in the crystal structure of nanoparticles by contrast to macroscale crystals, to the increased proportion of the nonautonomous phase atoms being under conditions other than those of the volume phase atoms (another coordination number, another symmetry of the local environment, the absence or other pattern of the long-range order), as well as the non-equilibrium nanoparticle state evolving due to extreme synthetic conditions, affect the properties of nanomaterials. In particular, there is a change in the following properties: mechanical - increased solidity in combination with high plasticity; electrical - small particles becoming semiconductors; magnetic - extreme dependence of magnetic properties on the particle size, with the transition to superparamagnetism; thermal lowered sintering temperatures, changed temperatures of polymorphic transformations due to high proportion of the liquid-like nonautonomous phase; optical - changed radiation and absorption spectra; chemical - increased reactivity, etc. [115,201-216]. As follows from the findings of a number of studies on the nanocrystals formation, their structure and properties are often governed by the chemical and thermal background of the original compositions and by the their formation mechanism [59,95,97-99, 119-121].

Applying the concept of nonautonomous finite-thickness phases was promising not only in terms of describing the behavior and properties of synthetic materials, but also in terms of analyzing natural systems. Previous studies $[29,215]$ illustrate the role of nonautonomous phases in mineral formation and their influence on properties of minerals. Such phenomena related to the nonautonomous phase properties were discovered as surface 
nanofragmentation, evoking fractality of nanocrystalline structures and absorption of the elevated impurity element concentrations, both incompatible with the autonomous phase structure in the crystal-chemical sense and compatible (isomorphic) impurities. The possibility to describe the volume phase interfaces as nonautonomous phases of finite thickness, which transition temperature to a liquid (liquid-like) state is lower than the melting point of the volume phase of the same composition, made it possible to explain many features of the ice behavior $[113,126,129]$.

Despite the above-mentioned examples of successful use of the concept of nonautonomous finite-thickness phases in terms of equilibrium (local-equilibrium) thermodynamics, this approach is quite limited and cannot always provide an adequate thermodynamic description of how the actualized structures are formed or identify conditions of their self-organization and methods to control interaction of disproportionate structural substance elements in the material. It is necessary to involve the methods of non-equilibrium thermodynamics and physical-chemical kinetics, along with further development of model concepts of disproportionate structural element interaction. Herewith, along with kinetic characteristics, the entropy indicators of internal conversional instability of energy related to the increasing role of conjugated thermodynamic flows and forces, to the emergence and maintenance of organized non-equilibrium states as so-called "dissipative" space-time structures of the substance are used for thermodynamic evaluation of non-equilibrium in ongoing processes and conditions of self-organization of these systems [217]. It is shown, as exemplified by [218], that in two-phase media with ongoing dissipative processes, the fluctuations of their characteristics become more favorable energetically than stationary conditions, providing smaller energy dissipation (entropy production). Increased parameters of the system state, while approximating the critical point, lead to abnormal growth of large-scale density fluctuations and the development of thermodynamic substance instability the phenomenon which is theoretically described based on the idea of "scale invariance (scaling)" to characterize the thermodynamic potentials by generalized homogeneous functions of the non-integer (fractional) order relative to their fields [219-221], and its practical application makes it possible to disperse materials up to nanoparticles under the influence of powerful energy flows, in particular, with the electric conductor explosion (ECE), experiencing the aggregate state transition through "solid-liquid-gaseous-plasma" [222]. There are great prospects for developing this line if ECE would be implemented in various chemically active media, in particular, in supercritical aqueous fluid to produce nanoscale metal oxides with a high proportion of the substance in the nonautonomous phases [223]. The arising objectives to describe the kinetics of heat and electricity transfer in various components to such a system (metal, liquid, vapor, plasma, supercritical fluids) are usually considered through the ideas of percolation theory and models of effective coefficients of inhomogeneous media [224,225]. They are built with aprioristic ideas about the typical sizes of heterogeneities, their mutual arrangement, the volume concentration, the nature of mutual contacts, etc. At the same time, the relationship between the transfer coefficients of each component and the characteristics of physical state of the substance (usually, the temperature and density) are assumed to be known. It is important to highlight that for liquid-metal and plasma media, the transfer coefficients are increasingly governed by the interaction between free electrons and small-and large-scale density fluctuations, along with temperature increase and approximation to the critical point of the substance [226]. In the vicinity of the critical point, this factor can be decisive for the calculation of effective transport coefficients of inhomogeneous medium and requires detailed consideration. Model concepts for the interaction of disproportionate substance structures are exemplified by the supramolecular model of eutectic [227,228] where, in contrast to the classical supramolecular ensembles [229], it is possible to violate the principle of geometric and topological complementarity, that is, the mutual consistency between the substrate and the receptor, including at the level of formation of intermolecular bonds [230,231]. The results of calculations performed for one-dimensional approximation within the classical Frenkel-Kontorova statistical model [232], as presented in this paper, demonstrate that suprastructures may organize themselves out of disproportionate substance structures and show that the probability of fragmentation for nonautonomous metastable suprastructures increases dramatically with an increase in the initial disproportion and with the formation of sufficiently strong intermolecular bonds. With due account to the above-mentioned significantly distinguishing properties of the substance in such "nonautonomous states" ("nonautonomous phases"), there are certain prospects for production of new functional materials with a high concentration of nonautonomous phases which use the features of metastable and intermediate structures formed as a result of these interactions, such as in the experimental material of the lithium-ion battery cathode [233]. However, there are still pending issues related to the approach of nonautonomous suprastructures to relaxation.

Research in the field of synthesis of new compounds with a high content of nonautonomous phases is aimed at both producing original functional materials with variable performance properties and developing related technologies for their production. In this regard, the synthesis of nanoceramics and other materials of constructional and functional purpose $[3,4,7,8,24,76,192,234-246]$, in particular, materials intended for use in thermoelectric energy converters [240,241], ferrites [13,25, 52, 59, 64,97-99, 107, 145], high-temperature superconductors [175, 238, 244], 
catalysts $[13,22,36,37,39,40,46,60]$, radioactive waste immobilization matrices $[236,237,239]$, is of great interest among the developments noted in Section 2.

The analysis confirms the scientific and practical relevance and prospects of the follow-up studies on thermodynamics and kinetics of nonautonomous phase formation and transformation both to identify the fundamental laws of interaction between macro-, micro - and nano-structural substance units and to produce new materials with a large proportion of nonautonomous phases with variable functional properties.

\section{Thermodynamics of the systems which include nonautonomous interphase areas and conjugated macroscale substance volumes}

In thermodynamic analysis, the nonautonomous phase is represented as an intermediate small-size interphase region with a typical sub-nanometer to nanometer thickness at relatively low temperatures which separates the surrounding macroscopic volume phases, different in composition, properties, and structure, through the open boundaries of which the system can exchange energy and substance. The forces of interatomic and intermolecular interaction manifested at these boundaries are macroscopically characterized by the surface tension, the value of which in the area of interface will vary in the direction of transition from one volume phase to another, and remain the same for all directions in the area of volume phases. Thermodynamic characteristic of the work link between the forces of surface tension $\sigma$, with the change in internal energy $d U$ and other parameters of the open system of variable composition, in the formation of interface region with the volume $d V_{f}$ and the flat surface area $d A_{f}$, is the combined ratio of the first and second laws of thermodynamics $d U=T d S-P d V_{f}+\sigma d A_{f}+\sum \mu_{j} d n_{j}$, where $P$ is the external pressure of the system, against which the interface region is formed; $d S$ is the system entropy variation during nonautonomous phase formation at a temperature $T ; \mu_{j}$ and $n_{j}$ are chemical potential and the number of moles of the $j$-th component in case of variations in the interphase substance content in the mass transfer. In case of the liquid or gaseous substance state and the curved interface, the pressure in the interfacial region will exceed the external pressure by the value of Laplace additional pressure $P_{L}=\sigma\left(1 / R_{1}+1 / R_{2}\right)$, where $R_{1}$ and $R_{2}$ are the curvature radii of two mutually perpendicular sections of the meniscus [247]. A large number of different experimental methods to measure directly the surface tension have been developed for these media, in contrast to solids where this is not possible, except for a few special cases, and calculation methods based on different models to describe the solid surface and surface energy are used to measure the surface tension (see, for instance, [248]).

\subsection{Equilibrium states of nonautonomous phases}

The thermodynamic equilibrium states and quasi-stationary equilibrium processes are usually characterized by well-known thermodynamic functions. Their definitions for the macroscopic volume phases of constant composition are given as conventional expressions of enthalpy $H=U+P V$, the variation of which governs by the thermal effect of isobaric process, Helmholtz free energy $F=U-T S$, the variation of which governs a part of internal energy that can be converted to the external work of the system in an isothermal process, Gibbs free energy (enthalpy)

$$
G=H-T S=U+P V-T S,
$$

the variation of which governs the total enthalpy and entropy effects, and the total system work minus expansion in isobaric-isothermal processes, as well as other thermodynamic potentials depending on the set of parameters used to describe the system state [249]. However, for the interphase region with an open interface, these functions are also related to the work of surface tension forces and the work of mass transfer forces $[110,111]$. Thus, from the differential form of the above definitions of thermodynamic functions

$$
\begin{gathered}
d H=d U+P d V+V d P, \quad d F=d U-T d S-S d T, \\
d G=d H-T d S-S d T=d U+P d V+V d P-T d S-S d T,
\end{gathered}
$$

based on the core equations for internal energy, it follows that the net mechanical system work

$$
d W=-P d V+\sigma d A_{f}=-[d F]_{T, n i},
$$

related to the changing volume and surface of the interphase region under conditions of constant temperature $(d T=0)$ and at constant substance content $\left(d n_{j}=0\right)$ is determined by the decrease of Helmholtz free energy. Herewith, the respective variation of the internal energy $d U$ causes an increase in the system entropy $d S=$ $(d U-d W) / T$. Integration of these equations at constant values of intensive parameters (i.e. not dependent on the mass of the system) according to the Euler theorem on homogeneous functions results in the following expressions 
for thermodynamic potentials of the interphase region, different from their usual expressions for volume phases due to the presence of an additional summand $\left(\sigma A_{f}\right)$ :

$$
\begin{gathered}
U=T S-P V_{f}+\sigma A_{f}+\sum \mu_{i} n_{i} ; \quad H=T S+\sigma A_{f}+\sum \mu_{i} n_{i} ; \\
F=-P V+\sigma A_{f}+\sum \mu_{i} n_{i} ; \quad G=\sigma A_{f}+\sum \mu_{i} n_{i} .
\end{gathered}
$$

This illustrates that in the region of phase interface, changes in the extensive properties will result in specific expressions for partial thermodynamic quantities (substance properties), as well as their ratios to the normal integral properties, in particular, for chemical potentials and their ratios to the Gibbs thermodynamic potential as a corollary of the Gibbs-Duhem equation. Similarly, the Gibbs-Duhem equation itself will differ, which links the intensive (system mass dependent) parameters for each phase of the multicomponent heterogeneous system

$$
S d T-V d P+A d \sigma+\sum n_{i} d \mu_{i}=0
$$

where $n_{i}$ is the number of particles of the $i$-th component, given their different phase state. This ratio for the phase interface region, at constant temperature $T$ and pressure $P$ values, becomes $A d \sigma=\sum n_{i} d \mu_{i}$, from which the Gibbs adsorption isotherm equation $d \sigma=\sum\left(n_{i} / A\right) d \mu_{i}$ is derived easily.

Although the known classical expressions given here show significant differences in the equilibrium thermodynamics of volume and surface phases, however, the local-equilibrium approach used is not able to reveal their self-organization and stability conditions in deterministic and spontaneous perturbations of thermodynamic quantities in non-equilibrium thermodynamic systems, which usually requires the use of physical-chemical kinetics methods. At the same time, the internal entropy production and internal instability values related to the increasing interaction of conjugated thermodynamic flows and forces, to the emergence and maintenance of organized non-equilibrium (metastable) states as so-called "dissipative" spatiotemporal structures of the substance, may be effectively used along with kinetic characteristics in order to evaluate thermodynamic the non-equilibrium of ongoing processes and self-organization conditions in such perturbed systems [217].

\subsection{Behavior of nonautonomous phases in non-equilibrium conditions}

In contrast to the above commonly accepted approach, the behavior of nonautonomous phases, as well as that of macroscopic volume phases, in non-equilibrium conditions is convenient to be considered based on the analysis of the system entropy alteration caused by irreversible processes, with the assistance of the extreme principles. Using the accepted system energy partition as heat and work during its exchange with the external environment, which is known to be

$$
d U=d Q+d W+\sum n_{i} d \mu_{i}
$$

for open systems, as well as the division of entropy variations, as proposed by I. Prigozhin [217], by the sum of two summands $d S=d_{e} S+d_{i} S$, one of which $\left(d_{e} S\right)$ is determined by the substance and energy exchange with the external environment, and the other $\left(d_{i} S \geq 0\right.$, always positive) by internal production due to irreversible processes in the system itself, the following is revealed for various conditions on its boundaries of nonautonomous phases.

1. If there is absolutely no energy and substance exchange, the state of the system, including the volume phases and interphase regions, as known for any isolated thermodynamic system, will tend to the equilibrium state with a maximum entropy value $S>S_{\max }$, which is determined by the growth of $d_{i} S \geq 0$ at $d_{e} S=0$ due to internal irreversible processes.

2. The $d_{e} S$ value is equivalent to the Clausius entropy variation, i.e. $d Q=T d_{e} S$, in case of the energy exchange only and with no substance exchange $\left(\sum \mu_{j} d n_{j}=0\right)$ on the boundary of the system considered, then the energy change is determined by the general expression

$$
d U=T d_{e} S+d W
$$

Given the ratio $d S=d_{e} S+d_{i} S$, it transforms as

$$
d U=T d S+d W-T d_{i} S
$$

and as

$$
d U=T d S-P d V+\sigma d A_{f}-T d_{i} S
$$

after substituting the above expression for work

$$
d W=-P d V+\sigma d A_{f}
$$

related to changes in the volume and surface of the interfacial region. 
It follows that in the conditions of constant temperature $T$, volume $V$ and surface $A$ of the interfacial region considered, its Helmholtz free energy $F=U-T S$ will evolve to the minimum value

$[d F]_{T, V, n_{i}}=\left[d U-T d S-S d T=d U-T d_{e} S-T d_{i} S=d Q-T d_{e} S-T d_{i} S-P d V+\sigma d A_{f}\right]_{T, V, n_{i}}=-T d_{i} S \leq 0$, and its Gibbs free energy (enthalpy)

$$
\begin{array}{r}
{[d G]_{T, P, n_{i}}=\left[d H-T d S-S d T=d U+P d V+V d P-T d S-S d T=-S d T+V d P+\sigma d A_{f}\right]_{T, P, n_{i}}} \\
=-T d_{i} S \leq 0
\end{array}
$$

will tend to the minimum in conditions of constant temperature $T$ and pressure $P$. Herewith, the internal energy

$$
[d U]_{S, V, n_{i}}=\left[T d s-P d V+\sigma d A_{f}\right]_{S, V, n_{i}}=-T d_{i} S \leq 0
$$

will tend to the minimum value in conditions of maintaining a constant value of total entropy $S$ by diverting its internal production to the external environment with $d S=0$ at $d_{i} S=-d_{e} S$, which is also a direct consequence of the second law of thermodynamics.

3. For open systems with external exchange of both energy and substance, these ratios are supplemented by the term $\left(\sum \mu_{j} d n_{j}\right)$ which factors into the input of the substance transfer (diffusion) and quantifies the above analysis of the system entropy variation, while preserving the extreme principles of thermodynamic potentials, the minima of which are also points (conditions) of stable mechanical and diffuse equilibrium. It is generally essential to take account of the thermal stability conditions, the expression of which is the enthalpy tending to minimum, with keeping the values $S, P, n_{i}$ :

$$
[d H]_{S, P, n_{i}}=\left[d U+P d V+V d P=T d S+V d P+\sigma d A_{f}+\sum \mu_{j} d n_{j}\right]_{S, P, n_{i}}=-T d_{i} S \leq 0
$$

constant, as well as of the chemical stability conditions in case of chemical reactions in the system, which, however, are not related to the additional specific extreme. The last conclusion follows from the Duhem-Jougeau theorem: the systems resistant to diffusion are resistant to chemical reactions as well [249]. As can be seen, the system state change in externally non-equilibrium conditions, aimed at achieving a stable equilibrium under the principle of thermodynamic potential minima, is related to the positive entropy production $d_{i} S \geq 0$ within the system itself due to irreversible processes occurring therein, which, however, occurs in isolated systems, thus determining achievement of their maximum entropy.

\subsection{Stability of non-equilibrium states}

Stability conditions of non-equilibrium systems can be considered on the same uniform basis, including of those with deterministic and spontaneous fluctuations of thermodynamic quantities and self-organization of dissipative regimes and structures. Herewith, the quadratic form $d_{i} S=\sum J_{K} d X_{K}$ is used as a common initial expression to describe entropy production in all irreversible processes according to [217]. It follows from the concept of entropy alteration in each irreversible process as the $d X_{K}$ thermodynamic forces (gradients of thermodynamic potentials) multiplied by the $J_{K}$ thermodynamic flows generated by them, for instance, the $d Q$ heat flow under the action of the difference between thermal potentials $\left(1 / T_{1}-1 / T_{2}\right)$, the $d n$ substance flow under the action of the difference between chemical potentials $\left(\mu_{1}-\mu_{2}\right)$ etc. In this case, the synergetic principle of least energy scattering (dissipation), equivalent to Prigozhin's theorem on minimum entropy production in stationary linear systems, which was previously used heuristically in the analysis of limits of energy conversion in high-power facilities, may be assumed as a thermodynamic stability condition [218].

As shown in these papers, it is convenient to use the relative non-stationarity coefficient of the $\varepsilon=$ $\left\langle E\left(J_{K} X_{K}\right)\right\rangle / \dot{E}\left(J_{0 K} X_{0 K}\right)$ form in order to generally estimate how dynamic perturbations of flows and forces effect the change in entropy production, as compared to their stationary values, and to identify the self-organization parameters on this basis. Herein, $E^{\prime}\left(J_{0 K} X_{0 K}\right)=T S^{\prime}$ is the value of energy dissipation (entropy production $\left.S^{\prime}\right)$ in the system with stationary values of flows and forces, $\left\langle E\left(J_{K} X_{K}\right)\right\rangle$ is the averaged value of this quantity in the system with perturbed flows and forces, determined by the standard rules of averaging. The value $\varepsilon=1$ corresponds to the unperturbed stationary state (mode) of the non-equilibrium system; the values $-1>\varepsilon>+1$ to the increased or reduced dissipative energy losses at various deviations from this mode. The mathematical formulation of the problem of mode stability and energy-entropy self-organization in this system is reduced to finding the lowest entropy generation value $\varepsilon=\varepsilon_{\min }$, depending on the parameters of disturbances. This is quite justifiable even for the systems which suppose a linear relation between thermodynamic flows and forces $J_{K}=\sum L_{K i} X_{i}$, 
in view of the general quadratic (bilinear) function of entropy production from the current flows and forces. In particular, the perturbation coefficient will be:

or

$$
\varepsilon=\frac{L_{11}\left\langle X_{1}^{2}\right\rangle+2 L_{12}\left\langle X_{1} X_{2}\right\rangle+\left\langle X_{22}\right\rangle}{L_{11} X_{01}^{2}+2 L_{12} X_{01} X_{02}+L_{22} X_{02}^{2}},
$$

$$
\varepsilon=\frac{\left\langle J_{1} X_{1}\right\rangle+\left\langle J_{2} X_{2}\right\rangle}{J_{01} X_{01}+J_{03} X_{02}}
$$

if addressing the energy and substance flows jointly. Since perturbations generally represent an overlay (superposition) of deterministic and random processes, both options have been analyzed. The time averaging is chosen from the two equal averaging variants represented by the ergodic hypothesis of equality between average time and average statistical values of the functions, i.e. $\langle\dot{E}\rangle=\bar{E}$, where

$$
\langle\dot{E}\rangle=\lim (1 / \tau) \int_{0}^{\infty} E(\tau) d \tau, \quad \bar{E}=\int_{-\infty}^{+\infty} E \psi(E) d E
$$

in case of deterministic periodic perturbations with one realizing function $E(\tau)$ and the statistical averaging $\bar{E}$ by the general aggregate or assemblage of random functions in case of random perturbations. Accordingly, $\tau$ is the period of oscillations; $\psi(E)$ is the density of the distribution probability of random disturbances. Thus, in the event of deterministic periodic oscillations of flows and forces, which can be considered as a harmonic process with random values of amplitude, frequency and phase $X_{i}(\tau)=X_{0 i}\left[1+n \sin \left(\omega_{i} \tau+\nu_{i}\right)\right]$, the averaged values will be: $\left\langle X_{i}(\tau)\right\rangle=X_{0 i}^{2}\left[1+n_{i}^{2} / 2\right],\left\langle X_{1}(\tau) X_{2}(\tau)\right\rangle=0$ at $\omega_{1} \neq \omega_{2}$ and $X_{01}=X_{02}\left[1+n_{1} n_{2} \cos \left(\nu_{1}-\nu_{2}\right) / 2\right]$ at $\omega_{1}=\omega_{2}$. According to the structure, these expressions are completely identical to the averaging results if perturbations of flows and forces are a purely chaotic fluctuation, for which the autocorrelation function of relation between two consecutive values in time is the $\delta$-Dirac function, and the probability of distribution density of $\psi(X)$ random deviations from the average statistical value according to the central limit theorem of the probability theory is expressed as a normal Gaussian distribution law of corresponding dimension.

Similar results are obtained when it comes to any other laws of probability distribution for random deviations of variables from the statistically average values, since the averaged values $X_{1}^{2}(\tau), X_{2}^{2}(\tau), X_{1}(\tau) X_{2}(\tau)$ are quadratic functions, and the respective characteristics of their scattering are central moments of the second order, from the definition of which these structures result directly.

Minimization of expressions for $\varepsilon$ leads to the identification of three successive system self-organization modes delimited by the roots of characteristic equation $\partial \varepsilon / \partial\left(n_{1} / n_{2}\right)=0$. As follows from this analysis, the condition required for energetically efficient self-organization of the thermodynamic systems under consideration, along with achieving the minimum entropy production $\varepsilon=\varepsilon_{\min }$ in the range of $\varepsilon_{\min } \leq 1$, is the antiphase $\left(\nu_{1}-\nu_{2}\right)=\pi$ feedback between the input and output flows (forces) carried out through forward and crossed conduction coefficients $L_{K i}$. However, the value of this relation in linear systems with the disturbances considered is insufficient to compensate for dissipative energy losses, except for extremely conjugate systems with $q=$ $L_{21} /\left(L_{11} L_{22}\right)^{1 / 2}=1$, where the maximum amplitude values of antiphase oscillations $n_{1}, n_{2}=1$ can be set at the same frequencies $\omega_{i}=\omega_{j}$. However, the conditions essential and sufficient for internal instability in linear systems can be realized at lower $q$ values if both flows and forces are simultaneously exposed to disturbances. The amplitude-phase characteristics of their oscillations are set in the range of $0.5 \leq \varepsilon \leq 1.5$ where the maximum value $\varepsilon_{\max }=1.5$ corresponds to the mode of in-phase oscillations of flows and forces $\left(\nu_{1}-\nu_{2}\right)=0,\left(\nu_{3}-\nu_{4}\right)=0$, and the lowest value $\varepsilon_{\min }=0.5$ to the mode of antiphase oscillations $\left(\nu_{1}-\nu_{2}\right)=\pi,\left(\nu_{3}-\nu_{4}\right)=\pi$ with maximum values of their amplitudes. The negative correlation coefficient

$$
\rho_{i j}= \pm \frac{\left\langle X_{i}(\tau) X_{j}(\tau)\right\rangle-\left\langle X_{i}(\tau)\right\rangle\left\langle X_{j}(\tau)\right\rangle}{\sigma_{i} \sigma_{j}},
$$

where $\sigma_{i}=\left[\left\langle X_{i}^{2}(\tau)\right\rangle-\left\langle X_{i}(\tau)\right\rangle^{2}\right]^{1 / 2}, \sigma_{j}=\left[\left\langle X_{j}^{2}(\tau)\right\rangle-\left\langle X_{j}(\tau)\right\rangle^{2}\right]^{1 / 2}$ are dispersions of variables, with chaotic spontaneous fluctuations of thermodynamic flows and forces meets the latter condition.

The nature and areas of self-organization are more diverse in thermodynamic systems when taking account of nonlinear effects of the transfer at different levels - from the simplest quasi-linear relation between phenomenological coefficients and one or more forces (flows), to the most complex cases of highly nonlinear systems with oscillations of the dissipative function. However, the analysis of the resulting ratio does not reveal fundamental differences from the results presented. This is explained as follows. Although the numerator expression for the relative non-stationarity coefficient $\varepsilon$, allowing for nonlinearities, transits from quadratic forms characteristic of linear and quasi-linear systems with one or two real roots of the characteristic equation which delimit the self-organization 
area, to forms of higher order which suppose a greater number of real roots and, accordingly, a greater number of self-organized states (modes), the number of positive roots that have a physical interpretation in the delimitation of modes does not increase. The latter is attributed to the fact that the number of sign reversals remains unchanged in the sequence of coefficients of the power polynomial in the expression for the non-stationarity coefficient when determining the dissipative function as accepted, in all cases of systems with a constant number of freedom degrees. Thus, in accordance with the Descartes rule, the uniform limit number of real positive roots of the polynomial is set and, therefore, the uniform global character of behavior of these systems is established with possible local patterns of nonlinearities in the range of complex roots.

\section{Heat-and-mass transfer and kinetics of chemical reactions in materials with a high content of nonautonomous phases}

A large content of nonautonomous phases is inherent in nanostructured materials, a formational characteristic of which are fractal self-organization processes in non-equilibrium systems [250]. They determine the metricstatistical self-similarity of different-scale composite structures with non-Euclidean (fractional) relation between the rate of increase in the number of elements considered and the increase in their consideration scale. This holds true for almost all synthesis techniques and methods for nanomaterials and is determined by the special role of energy and entropy characteristics of surface layers, by the correlation scale of the substance and energy transfer in different-sized macro-, micro- and nanostructured material units, as well as by the tolerance factor of their interface. Given that the transition $(10-100 \mathrm{~nm})$ layer from volumetric $3 D$ material to the two-dimensional $2 D$ surface may include several subsurface zones with gradual decrease of substance filling in the range of $3>D>2$ at sub-monolayer $(0.5-1.5 \mathrm{~nm})$ non-stoichiometric zone at the phase interface with dimension $2>D>1$, or even $1>D>0$, its properties are governed by a set of physical phenomena of three-dimensional volume, two-dimensional surface, and in some cases even of one-dimensional and point elements. This approach reveals an extensive rank of self-similar transition structures of fractional dimension, in which the internal energy determining the different physical properties of the material, may significantly increase not only by increasing the binding energy and the number of these bonds in the crystalline lattice, but also due to the influence of electrical and chemical surface potential, as well as due to the growth of the entropy factor. At the same time, the kinetic and dynamic characteristics of nanocomposite materials also change significantly, since the energy accumulation level that determines the rate of degradation processes therein is limited by the surface zone thickness, and the energy dissipation has a significantly higher rate in the surface layer than in the volume. This is confirmed by experimental observations of phase transitions on the surface of solids, by measurements of electrical strength and thermal conductivity in the volume and on the surface [251-253]. As shown in [254], a condition for fractal self-organization of non-equilibrium systems is achieving the threshold value of energy dissipation, starting from which the system internal irreversible processes and exchange with the environment are accompanied by excess of entropy return in comparison with its generation. An essential condition for these processes is that there is non-linear feedback between the system and the energy source, as well as fluctuations and bifurcations in the transitions between individual states of the system until it becomes stable with minimal entropy production.

The noted fundamental aspects in fractal ordering of nanostructured materials with a high content of nonautonomous phases are taken into account in the developed models to calculate the heat and mass transfer and chemical kinetics (substance and energy transfer) occurring under spatial and mass limitations of nonautonomous phases. They are analyzed in fractal (fractionally dimensioned) space on the basis of modified Fick / Fourier law and nonlocal coordinate and time transfer equation for fractional order derivatives in the micro-layered medium [255,256]. Herewith, the transition from whole-order derivatives to fractional-order ones is realized according to the RiemannLiouville and Riesz-Weyl scheme after averaging the common equation of heat and substance propagation under the assumption that the thermal conductivity and mass transfer coefficients as random functions of the spatial coordinate in a micro-layered medium may be regarded as an integral operator. Its kernel in a statistically homogeneous medium is a product of Green's function which suits both the problem considered and the correlation ratio in averaging the initial equation. Given that the time and space intervals form the Hausdorff-Bezikovich set for the media of fractal structure with phenomena of spatial correlations and memory effect, this transition makes it possible to directly link the indicators of fractional derivatives with the respective ones of fractal dimension in time and space. As a result, without resorting to commonly used numerical methods (for instance, [257]), it is possible to derive the analytical model expressions for diffusion and temperature distribution problems [258], for instance, in classically shaped micro-inhomogeneous bodies (wires, films, plates, rods, bars, etc.), using wellknown constructive models of regular fractal media with a regular geometric structure (Cantor comb $D<1$; Koch snowflake $1<D<2$; Sierpinski sieve and fan, Menger square and sponge $2<D<3$ ), as well as walk models 
with fractal generalization of Einstein's relation. Their equivalent parameters are close enough to replace the real pseudo-regular structures with a fractal model.

The obtained solutions occupy an intermediate position between relaxation and wave behavior in time, and lead to a power dependence on spatial arguments, which is confirmed by the experimental data obtained [259,260]. Thus, in the stationary case compared to the linear temperature distribution in the continuous homogeneous medium layer, the nonlinear temperature distribution with a bulge downward is inherent in the fractal micro-inhomogeneous medium layer dimensioned less than one (Cantor comb), and with a bulge upwards (Fig. 2) in the environment dimensioned more than one (Koch snowflake, Sierpinski sieve, Menger sponge), which is generally consistent with experimental results.

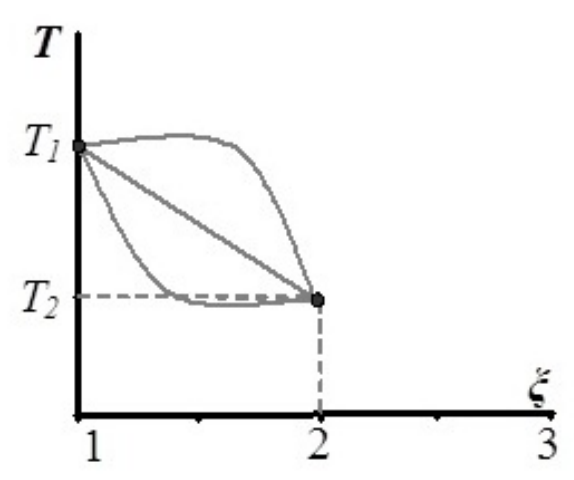

FIG. 2. Temperature distribution in a layer with a fractal structure: 1,2,3 - the nature of temperature distribution in a layer of a solid homogeneous medium, of a medium with a fractal dimension more or less than one, respectively [258]

However, it should be noted that this approach is sometimes insufficient to characterize the fractal structures of occasional nature, self-similarity of which cannot be adequately described using only one fractal (Hausdorff) dimension with a constant value, and which requires specifying the range of multifractal dimensions based on the generalized Renyi formula:

$$
D_{R q}=\lim _{\varepsilon \rightarrow 0} \lim _{\tau \rightarrow 0} \lim _{m \rightarrow 0}\left[\frac{1}{1-q} \frac{\ln I_{R q}(q, \varepsilon)}{\ln (1 / \varepsilon)}\right]
$$

Herein, $I_{R q}(q, \varepsilon)=\left[\sum_{i=1}^{M(\varepsilon)} p_{i}^{q}(\varepsilon)\right]$ is the generalized Renyi entropy of the $q$ order; $M(\varepsilon)$ is the minimum number of "measuring" cubes with an $\varepsilon$ edge required to cover the fractal in the $n$-dimensional phase coordinate space of the embedding; $p_{i}$ is for the probabilities of visiting the $i$-th cube by the phase trajectory; $m$ is the number of points used to estimate the dimension [261]. Known expressions for the Kolmogorov-Sinai entropy and KolmogorovHausdorff fractal dimension $(q=0)$, Shannon entropy, and the corresponding information dimension $(q=1)$, correlation entropy, and correlation dimension $(q=2)$ follow from this relation as special cases at various $q$ [262]. The change in fractal dimension along the layer of strongly micro-nonhomogeneous material can result in nonuniformity of its thermal and physical characteristics, as experimentally recorded in [263], and, consequently, in undulating temperature distribution (Fig. 3).

The proposed approach helped circumvent the limits of traditional macroscopic models of transfer processes in composite systems with relatively small filler concentrations and with no nonlocal effects of the influence of structural element scale on the rate of the transfer process. They are generalized in the well-known reference book [265] and are based on the generalized conductivity principle tracing its origin to the papers by Poisson, Clausius, Mossotti, Lorenz, Lorentz Maxwell, Rayleigh, Wiener, Lichtenecker, Odelevskii, etc.

One of the important integral effects of multifractal media in composite materials with electro-dual (conductive and non-conductive) components is that the coefficient of effective conductivity that occurs at the percolation threshold during formation of a conductivity chain cluster is independent of the specific arrangement of components when it is determined only by the ratio between their concentrations and electrical characteristics. The solutions obtained for the above models confirm the known results for the model of A. M. Dykhne [266] who identified this relation as $\sigma_{e}=\left[\sigma_{1} \sigma_{2} / p(1-p)\right]^{1 / 2}$, where $\sigma_{1}$ and $\sigma_{2}$ are conductivity values of the components, $p$ and $(1-p)$ are their concentrations. 


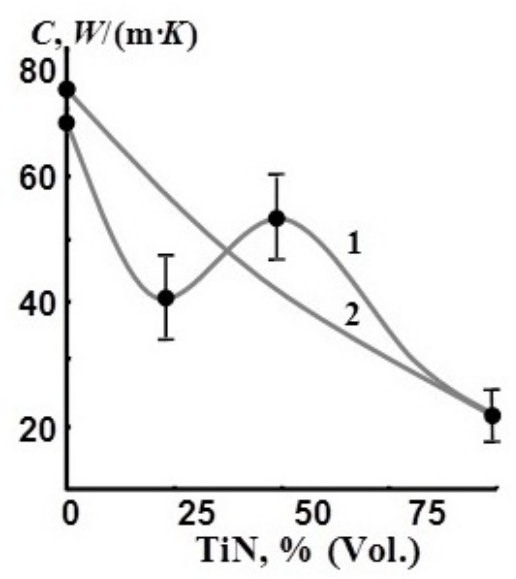

FIG. 3. Comparison of theoretical and measured values of thermal conductivity of sintered TiNAlN composite: 1 - thermal conductivity determined on the basis of measurements at $T=30{ }^{\circ} \mathrm{C}$; 2 - theoretical dependence for the "ideal" mixture of non-interacting components [264]

Modeling of chemical kinetics in the systems under consideration may also be carried out on the basis of nonequilibrium Prigozhin and Tribus's thermodynamic approach, using Janes's formalism for statistical interpretation of the results based on modern information theory in the stage-by-stage consideration of the processes presented in the experimental part of this review. These include the process of hydrothermal synthesis in autoclaves or in an electric underwater conductor explosion which is used to produce oxide nanopowders during high-temperature hydrolysis of precursor salts in the aqueous supercritical-pressure fluid capable of dissolving substances that are almost insoluble under normal conditions. The initial $p H$ value of the medium, the synthesis duration and temperature, the system internal pressure value are basic parameters determining both the kinetics of the processes and the properties of the resulting products. Since the major cause of homogeneous particle aggregation, related to the surface tension at the phase interface, is absent under supercritical conditions, the supercritical water is able to dissolve substances which are almost insoluble under normal conditions, including some oxides [267-270]. Subsequently, the solid crystalline phase of oxide nanoparticles, of nearly the same size and with a fairly developed surface, is released if creating conditions for the solution oversaturation. The critical degree of oversaturation $\gamma=c / c s$ can be estimated by expressing the above chemical potential difference $\Delta \mu=2 \sigma(\infty) V a / R_{c r}$ of the critical nanoparticle nucleus and the phase-forming medium through the substance concentration in the supersaturated solution and its equilibrium solubility $c s$ relative to the crystalline phase. This results in

$$
\ln \gamma=\ln \left(\frac{c}{c s}\right)=\frac{\Delta \mu}{R T}=\frac{2 \sigma(\infty) V a}{R_{c r} R T}
$$

for the perfect solution. The critical size of the spherical nucleus $R_{c r}$ results from the condition of the maximum $d(\Delta G) / d R=0$ of the Gibbs free energy defined as the sum of its volume and surface components

$$
\Delta G=-\frac{4}{3} \pi \frac{R^{3} \Delta \mu}{V a}+4 \pi R^{2} \sigma(R)
$$

According to [271], it is

$$
R_{c r}=\frac{2 \sigma(\infty) V a}{\Delta \mu}
$$

without factoring into the relation between the surface tension and the particle size, and

$$
R_{c r}=l_{0}\left[1-\frac{2 \delta}{l_{0}}+\left(\frac{2 \delta}{l_{0}}+1\right)^{1 / 2}\right]
$$

if it is factored into at small particle radii on the ratio $\sigma(R) \approx \sigma(\infty) /(1+2 \delta / R)$ where $l_{0}=\sigma(\infty) V a / \Delta \mu, \delta$ is the "tolmen length". The critical Gibbs energy at the maximum point during formation of homogeneous condensation nuclei on the surface of the nuclei emerging spontaneously as a result of fluctuations in the density and concentration of the substance in the system, without regard to the relation between the surface tension and the particle size, will be $\Delta G_{c r}=(4 / 3) 2 \pi R_{c r}(\sigma-2 / 3 \sigma)$, or a third of the surface energy of the nucleus. The remaining two-thirds are compensated by the chemical energy component determined by the energy benefit of phase transition. Herewith, 
$\Delta \mu=2 \sigma(\infty) V a / R_{c r}$. Similarly, it is possible to make quantitative estimates for heterogeneous condensation in the new phase formation on existing surfaces (vessel walls, foreign substance particles - condensation nuclei), as well as it is also possible to estimate using the equation given in the liquid phase during solution supercooling, in view of the known relation $\Delta \mu=\Delta H_{n}-T \Delta S_{n}=\Delta H_{n} \Delta T / T_{n}$, where $\Delta T=\left(T_{n}-T\right)$ is supercooling, $\Delta H_{n}$ is the process enthalpy. These estimates show that the work of formation of solid nuclei at heterogeneous condensation will be as many times less than the work of a homogeneous process as the volume of the drop nucleus on the surface of the condensation nucleus is less than the volume of the sphere of the same curvature. These estimates relate only to a single nucleus and do not factor into the entropy component of the formation energy in the event of mass of nuclei. The entropy component can compensate the surface energy and provide spontaneous dispersion at low values of interfacial tension. Estimation of nucleus formation conditions in the nonautonomous substance state, [272-287], i.e. the substance being under spatial limitations that do not allow the formation of a critical nucleus of a new phase, in particular, due to the nanoparticles of another phase distributed in these media, is significantly complicated and requires factoring into the structure, composition and properties of the latter [106]. In case of classical nucleus forms (cube, ball, cylinder), it is possible to derive analytical expressions of the relation between their geometric characteristics and the size of the new-phase nucleus [105]. The kinetics of condensation formation of a new phase in hydrothermal synthesis, according to M. Folmer and J. I. Frenkel theory [288, 289], is defined as the rate of occurrence of condensation centers $I=A_{1} \exp \left[-\Delta G_{1} /(R T)\right]$ and the rate of the substance transfer to the resulting condensation centers $U=A_{2} \exp \left[-E_{\eta} /(R T)\right]$. The first of them is proportional to the probability of the condensation centers origin according to Gibbs energy $\Delta G_{1}$, and the second is proportional to the probability of their "survival" according to the activation energy $E_{\eta}$ of the substance transition from the initial phase to the nucleus surface. The total rate is determined by the product of these probabilities and can show an extreme, depending on the ratio between the energies $\Delta G_{1}, E_{\eta}$, the temperature $T$ and the proportionality coefficients $A_{1}, A_{2}$.

The applicability of the general thermodynamic approach to the estimation of homogeneous formation of small particles is limited by the condition of a significant excess of the specific surface energy

$$
\sigma(R) \approx \frac{\sigma(\infty)}{1+2 \delta / R}
$$

over its mean-square fluctuations

$$
\langle\langle\Delta \sigma\rangle 2\rangle=\frac{k T}{8 \pi R}\left(\frac{\delta \sigma}{\partial R}\right)_{P, S}=\frac{k T \delta \sigma(\infty)}{4 \pi R(R+2 \delta)^{2}} .
$$

This is done in the case of $R \gg[k T \delta / \sigma(\infty)]^{1 / 3}$. Estimates show that the critical nucleus size may be $R_{c r}=(5 \div 10) \cdot 10^{-10} \mathrm{~m}$ at a low condensation temperature, that is, be sized as several atoms. In this case, $\Delta G$ will vary discretely when the next atom joins, which is incompatible with the thermodynamic theory assumption of continuous change of surface energy and Gibbs free energy. It is essential to shift towards the statistical nucleation theory, based on the interaction parameters for individual atoms and their agglomerates, for example, for Arrefyllius phases.

The kinetic features of nanomaterials production reactions considered in the experimental section of this review, for example, of nanomaterials produced on the basis of solid-phase synthesis technologies or solid-flame reactions of self-propagating high-temperature synthesis where combustion proceeds in the mode of reagent diffusion through a layer of solid products formed, are described satisfactorily enough by the above fractal-diffusion approach. The relationship between the traditional macroscopic characteristics of chemical kinetics and statistical interpretation of thermodynamic reaction parameters based on modern information theory is used for the model evaluation of the rate performance of chemical reactions in the nanomaterial synthesis by gas-flame combustion, occurring in the kinetic mode with the evaporation of solid or liquid reagents, as well as of the kinetics of their interaction with other chemicals. Thus, according to Eyring [290], under the assumption that the reaction rate $r^{\prime}$ in a chemically interacting system is proportional to the number of particles $N_{i}$ with sufficient threshold energy $E_{i}$, where the total number of particles is $N$ in the system, it is possible to derive well-known Arrhenius's ratio $r^{\prime} \approx N \exp \left(-\beta E_{i}\right)$ after minimizing Shannon's expression for the information entropy $S=-k \sum p_{i} \ln p_{i}$ with additional conditions imposed on the sum of the probabilities of all possible particle states and the mathematical expectations of the macroscopic characteristics of the system. It is also possible to link

$$
\frac{\partial\left(\ln r^{\prime}\right)}{\partial(1 / k T)}=-E_{i}=-\frac{\Delta E}{N}
$$




$$
\frac{\partial\left(\ln r^{\prime}\right)}{\partial(1 / T)}=-\frac{\Delta E}{R}
$$

out of the Boltzmann distribution for the kinetic energy of molecules by its logarithm and differentiation by $\beta=1 / k T$, where $\Delta E$ is the activation energy. The resulting expression is of similar form with the known equation describing the change in the equilibrium constant with that in the temperature and enthalpy of the components, and the change in the free Gibbs energy $G=U+P V-T S$ through it, and then the change in the proportion of the reacted particles $\alpha=-\mu / R T$ of their total $N_{i}$. Herein, Gibbs chemical potential $\mu$ and Gibbs specific free energy (per particle) are coincident in case of the pure substance, while Gibbs free energy is defined as the sum of all components $G=\sum(\mu N)$ for all elements in case of the substance mixture.

The relationships presented also make it possible to determine the concurrent entropy variation $S$ attributed to the ongoing chemical reactions and to assess the behavior of nonautonomous phases in non-equilibrium conditions, as well as to analyze the overall system stability in accordance with thermodynamic criteria presented earlier, involving the extreme principles.

\section{Conclusion}

Analysis of the problem of designing new functional materials with variable performance characteristics indicates a great role in its solution of special small-size micro- and nanoscale objects formed as intermediate (transitional) structures in a limited space between the macroscopic volume phases and with a limited substance amount. They differ greatly in their properties, structure and composition of the relevant characteristics from the volume phases and cannot thermodynamically exist in isolation from them as autonomous phases. It distinguishes fundamentally the nonautonomous phase from autonomous ones. This also determines the features of thermodynamics and kinetics of interaction of disproportionate structural units of substance in the material, the conditions of their self-organization and control methods for such interaction in the material synthesis, as important in fundamental and applied terms, but not always considered in the development of material technology.

Studying the behavior and properties of nonautonomous phases requires the use of various methods and approaches, in particular, theoretical and experimental methods of nonequilibrium thermodynamics, chemical kinetics, nonlinear dynamics and fractal analysis. The results presented show that great opportunities for providing the required functional properties of materials are related to the use of nanocomposites, which, as a rule, include a significant proportion of nonautonomous phases. The unifying characteristic of their formation in nonequilibrium systems is that of fractal self-organization. They determine the metric-statistical self-similarity of different-scale structures with non-Euclidean (fractional) relation between the rate of increase in the number of considered elements and the increase in the scale of consideration. This holds true for almost all technologies and methods for nanomaterial synthesis and is determined by the special role of energy and entropy characteristics of nonautonomous phases, the correlation scale of substance and energy transfer in different-sized macro-, micro- and nanostructured material units, as well as by the tolerance factor of their interface. At the same time, kinetic and dynamic characteristics of nanocomposite materials are also significantly changed, since the energy accumulation level, which determines the rate of degradation processes therein, is limited by the size of the border zone where the rate of energy dissipation is significantly greater than that in the volume. Achieving the threshold value of energy dissipation, starting from which irreversible processes are accompanied by excess of entropy return over its generation inside the system and in its exchange with the environment, is a condition of structural self-organization realized if there is nonlinear positive feedback from the energy source. Bifurcation transition of nonequilibrium systems to a stable state with minimal entropy production can be facilitated by antiphase deterministic and chaotic spontaneous fluctuations of thermodynamic flows and forces with negative correlation coefficient.

These factors generically determine the structural and energy approach to improve the design of functional materials with variable performance properties based on the use of nanoscale reaction zones with ongoing heat and mass transfer, chemical and phase transformations both in a relatively slow diffusion mode of hydrothermal or solid-phase high-temperature synthesis and in a high-speed kinetic mode of precursor medium combustion. Experimental studies of the effect of phase composition heterogeneity on the change in functional properties and stability of nanocomposite materials with a large proportion of nonautonomous phases generally confirm these ideas.

Promoting the concept of nonautonomous phases and their genesis in the areas discussed above opens up certain reserves for solving the problems of physical and chemical design of new functional nanocomposite materials which consist largely of nonautonomous phases, for developing technologies for their synthesis and consolidation, which ensure saving of the individual properties of nanoscale objects in the system. 


\section{References}

[1] Almjasheva O.V., Gusarov V.V. The role of non-autonomous state in the formation of nanomaterials structure and properties. In Nanomaterials: Properties and Potential Applications, Moscow, Nauchnyi Mir, 2014, P. 384-409 (in Russian).

[2] Miyazaki C.M., Riul A. Low-Dimensional Systems: Nanoparticles. Nanostructures, 2017, 5, P. $125-146$.

[3] Rempel A.A. Nanotechnologies. Properties and applications of nanostructured materials. Russ. Chem. Rev., 2007,76 (5), P. $435-461$.

[4] Vance M.E., Kuiken T., et al. Nanotechnology in the real world: Redeveloping the nanomaterial consumer products inventory. J. Nanotechnol., 2015, 6 (1), P. 1769-1780.

[5] Byrappa K., Adschiri T. Hydrothermal technology for nanotechnology. Progress in Crystal Growth and Characterization of Materials, 2007, 53, P. 117-166.

[6] Kirillova S.A., Almjashev V.I., Gusarov V.V. Phase relationships in the $\mathrm{SiO}_{2}-\mathrm{TiO}_{2}$ system. Russ. J. Inorg. Chem., 2011, 56 (9), P. 14641471.

[7] Newnham R. Size effect and nonlinear phenomena in ferroic ceramics. Editrice Iberica, Faenza, 1993, 2, P. 1-9.

[8] Dahman Y. Nanoparticles (Chapter 5). Nanotechnology and Functional Materials for Engineers A volume in Micro and Nano Technologies, 2017, P. 93-119 p.

[9] Gleiter H. Nanostructured materials: basic concepts and microstructure. Acta Mater., 2000, 48 (1), P. 1-29.

[10] Kushnir S.E., Kazin P.E., Trusov L.A., Tretyakov Yu.D. Self-organization of micro- and nanoparticles in ferrofluids. Russian Chemical Reviews, 2012, 81 (6), P. 560-570.

[11] Ostroushko A.A., Russkikh O.V. Oxide material synthesis by combustion of organic-inorganic compositions. Nanosystems: Phys. Chem. Math., 2017, 8 (4), P. 476-502

[12] Egorysheva A.V., Kuvshinova T.B., et al. Synthesis of high-purity nanocrystalline BiFeO 3 . Inorganic Materials, 2013 , 49 (3), P. $310-314$.

[13] Popkov V.I., Almjasheva O.V., et al. Magnetic properties of $\mathrm{YFeO}_{3}$ nanocrystals obtained by different soft-chemical methods. Journal of Materials Science: Materials in Electronics, 2017, 28 (10), P. 163-170.

[14] Mahalakshmi S., Srinivasa Manja K., Nithiyanantham S. Electrical properties of nanophase ferrites doped with rare earth ions. J. Supercond. Nov. Magn., 2014, 27 (9), P. 2083-2088.

[15] Lomanova N.A., Gusarov V.V. Effect of surface melting on the formation and growth of nanocrystals in the $\mathrm{Bi}_{2} \mathrm{O}_{3}-\mathrm{Fe}_{2} \mathrm{O}_{3}$ system. Russ. J. Gen. Chem., 2014, 83 (12) P. 2251-2253.

[16] Khaliullin Sh.M., Zhuravlev V.D., Bamburov V.G. Solution-combustion synthesis of $\mathrm{MZrO}_{3}$ zirconates $(\mathrm{M}=\mathrm{Ca}$, Sr, Ba) in open reactor: thermodynamic analysis and experiment. International Journal of Self-Propagating High-Temperature Synthesis, 2017,26 (2), P. 93-101.

[17] Krasilin A.A., Gusarov V.V. Control over morphology of magnesium-aluminum hydrosilicate nanoscrolls. Russ. J. Appl. Chem., 2015, 88, P. $1928-1935$.

[18] Bandyopadhyay A.K. Nano Materials. New Age International (P) Ltd., Publishers, New Delhi, 2008,321 p.

[19] Arkhipov D.I., Osmolovskaya O.M., et al. Optimization of $\mathrm{CrO}_{2}$ magnetic characteristics in the process of hydrothermal synthesis using nucleating agents of complex structure. Russ. J. Gen. Chem., 2016, 86 (4), P. 983-985.

[20] Sadovnikov S.I., Rempel A.A., Gusev A.I. Nanostructured silver sulfide: synthesis of various forms and their application. Russ. Chem. Reviews, 2018, 87 (4), P. 303-327.

[21] Aruna S.T., Mukasyan A.S. Combustion synthesis and nanomaterials. Curr. Opin. Solid State Mater. Sci., 2008,12 (3-4), P. 44-50.

[22] Gonzalez-Cortes S.L., Imbert F.E. Fundamentals, properties and applications of solid catalysts prepared by solution combustion synthesis (SCS). Appl. Catal. A Gen., 2013, 452, P. 117-131.

[23] Karn B., Kuiken T., Otto M. Nanotechnology and in Situ Remediation: A Review of the Benefits and Potential Risks. Environ. Health Perspect, 2009, 117 (12), P. 1823-1831.

[24] Lee J., Mahendra S., Alvarez P.J.J. Nanomaterials in the Construction Industry: A Review of Their Applications and Environmental Health and Safety Considerations. ACS Nano, 2010, 4 (7), P. 3580-3590.

[25] Coutinho P.V., Cunha F., Barrozo P. Structural, vibrational and magnetic properties of the orthoferrites $\mathrm{LaFeO}_{3}$ and $\mathrm{YFeO}_{3}$ : A comparative study. Solid State Communications, 2017, 252, P. 59-63.

[26] L'vov P.E., Svetukhin V.V. Thermodynamics of the phase equilibrium of multicomponent solid solutions containing nano-sized precipitates of the second phase. Physics of the Solid State, 2013, 55 (11), P. 2374-2380.

[27] Xu D., Johnson W.L. Geometric model for the critical-value problem of nucleation phenomena containing the size effect of nucleating agent. Phys. Rev. B, 2005, 72, 052101.

[28] Khambaty S., Larson M.A. Crystal regeneration and growth of small crystals in contact nucleation. I\&EC Fundamentals, 1978 , 17 (3), P. $160-165$.

[29] Tauson V.L., Akimov V.V. Effect of crystallite size on solid state miscibility: applications to the pyrite-cattierite system. Gochimica et Cosmocimica Acta, 1991, 55 (10), P. 2851-2859.

[30] Murdoch H.A., Schuh C.A. Stability of binary nanocrystalline alloys against grain growth and phase separation. Acta Mater, 2013, 61 (6), P. 2121-2132.

[31] Shmyt'ko I.M., Ganeeva G.R., Aronin A.S. Influence of grain boundaries on the formation of new structural states in simple rare-earth oxides. Physics of the Solid State, 2015, 57 (1), P. 136-141.

[32] Tsoga A., Nicolopoulos P. Surface and grain-boundary energies in yttria stabilized zirconia (YSZ -8 mol \%). J. Mater. Sci., 1996, 31 (20), P. 5409-5413.

[33] Vasiliev A.L., Poyato R., Padture N.P. Singlewall carbon nanotubes at ceramic grain boundaries. Scripta Mater., 2007,56 , P. $461-463$.

[34] Ebeling W., Engel A., Feistel R. Physik der Evolutionsprozesse. Akademie-Verlag, Berlin, 1990, 371 p.

[35] Saber M., Kotan H., Koch C.C., Scattergood R.O. Thermodynamic stabilization of nanocrystalline binary alloys. J. Appl. Phys., 2013, 113, 063515.

[36] Rodionov I.A, Zvereva I.A. Photocatalytic activity of layered perovskite-like oxides in practically valuable chemical reactions. Russ. Chem. Rev., 2016, 85 (3) P. 248-279.

[37] Bugrov A.N., Rodionov I.A., et al. Photocatalytic activity and luminescent properties of Y, Eu, Tb, Sm and Er-doped $\mathrm{ZrO}_{2}$ nanoparticles obtained by hydrothermal method. Int. J. Nanotechnology, 2016, 13 (1/2/3), P. 147-157. 
[38] De Prado N.T., Oliveir L.C.A. Nanostructured niobium oxide synthetized by a new route using hydrothermal treatment: High efficiency inoxidation reactions. Applied Catalysis B: Environmental, 2017, 205, P. 481-488.

[39] Yang Q., Hu H., Wang S.S. Preparation and desulfurization activity of nano- $\mathrm{CeO}_{2} / \gamma-\mathrm{Al}_{2} \mathrm{O}_{3}$ catalysts. Rare Met., 2018, 37, P. 554-560.

[40] Al'myasheva O.V, Vlasov E.A., Khabenskii V.B., Gusarov V.V. Thermal stability and catalytic properties of the composite amorphous $\mathrm{Al}_{2} \mathrm{O}_{3}$-nanocrystals $\mathrm{ZrO}_{2}$. Russ. J. Appl. Chem., 2009, 82 (2) P. $217-221$.

[41] Livage J. Sol-gel synthesis of heterogeneous catalysts from aqueous solutions. Catalysis Today, 1998, 41 (1-3), P. 3-19.

[42] Li X., Tang C.J., et al. Controllable synthesis of pure-phase rare-earth orthoferrites hollow spheres with a porous shell and their catalytic performance for the CO+NO reaction. Chem. Mater, 2010, 22, P. 4879-4889.

[43] Grabowska E. Selected perovskite oxides: Characterization, preparation and photocatalytic properties - A review. Applied Catalysis B: Environmental, 2016, 186, P. 97-126.

[44] Iglesia E., Barton D.G., et al. Bifunctional pathways in catalysis by solid acids and bases. Catalysis Today, 1997, 38 (3), P. $339-360$.

[45] Chen X., Liang S.-J., et al. Self-propagating combustion synthesis of nanocrystalline yttrium iron oxide solid solution photocatalysts. Chinese J. Inorg. Chem., 2009, 25 (11), P. 1922-1927.

[46] Niu X.S., Li H.H., Liu G.G. Preparation, characterization and photocatalytic properties of REFeO 3 (RE = Sm, Eu, Gd). J. Mol. Catal. A: Chem., 2005, 232 (1-2), P. 89-93.

[47] Wu R., Xie P., et al. Hydrothermally prepared $\mathrm{Cr}_{2} \mathrm{O}_{3}-\mathrm{ZrO}_{2}$ as a novel efficient catalyst for dehydrogenation of propane with $\mathrm{CO}$. Catalysis Communications, 2013, 39, P. 20-23.

[48] Lee J., Jeon H., et al. Morphology-dependent phase transformation of $\gamma-\mathrm{Al}_{2} \mathrm{O}_{3}$. Applied Catalysis A: General, 2015 , 500, P. 58-60.

[49] Falconer K.J. Fractal geometry. Mathematical foundations and applications. John Wiley \& Sons, New York, 1990,337 p.

[50] Lau K.-S., Ngai S.-M. Multifractal measures and a weak separation condition. Advances in mathematics, 1999, 141, P. 45-96.

[51] Ni H., Zhang L., et al. Supercritical fluids at subduction zones: Evidence, formation condition, and physicochemical properties. EarthScience Reviews, 2017, 167, P. 62-71.

[52] Kefeni K.K., Msagati T.A.M., Mamba B.B. Ferrite nanoparticles: Synthesis, characterisation and applications in electronic device. Materials Science and Engineering: B, 2017, 215, P. 37-55.

[53] Ahmad T., Lone I.H., et al. Multifunctional properties and applications of yttrium ferrite nanoparticles prepared by citrate precursor route. Materials \& Design, 2017, 126, P. 331-338.

[54] Karouia F., Boualleg M., Digne M., Alphonse P. The impact of nanocrystallite size and shape on phase transformation: Application to the boehmite/alumina transformation. Advanced Powder Technology, 2016, 27 (4), P. 1814-1820.

[55] Zhuravlev V.D., Lobachevskaya N.I., et al. New Vanadium Germanium Garnets. Doklady Chemistry, 2018,479 (2), P. $45-48$.

[56] Nakayama S. $\mathrm{LaFeO}_{3}$ perovskite-type oxide prepared by oxide mixing, co-precipitation and complex synthesis methods. Journal of Material science, 2011, 36, P. 5643-5648.

[57] Hakuta Y., Hayashi H., Arai K. Fine particle formation using supercritical fluids. Current Opinion in Solid State and Materials Science, 2003, 7 (4-5), P. 341-351.

[58] Racu A.V., Ursu D.H., et al. Direct low temperature hydrothermal synthesis of $\mathrm{YFeO}_{3}$ microcrystals. Mater. Lett., 2015, 140, P. 107-110.

[59] Tugova E.A., Zvereva I.A. Formation Mechanism of $\mathrm{GdFeO}_{3}$ nanoparticles under the hydrothermal conditions. Nanosystems: Phys. Chem. Math., 2013, 4 (6), P. 851-856.

[60] Chislova, I.V., Matveeva A.A., Volkova A.V., Zvereva I.A. Sol-gel synthesis of nanostructured perovskite-like gadolinium ferrites. Glass Phys. Chem., 2011, 37 (6), P. 653-660.

[61] Mukasyan A.S., Rogachev A.S. Combustion synthesis: mechanically induced nanostructured materials. J. Mater. Sci., 2017, 52, P. 1182611833.

[62] Komlev A.A., Vilezhaninov E.F. Glycine-nitrate combustion synthesis of nanopowders based on nonstoichiometric magnesium-aluminum spinel. Russ. J. Appl. Chem., 2013, 86 (9) P. 1344-1350.

[63] Bubnova R.S., Firsova V.A., Volkov S.N., Filatov S.K. Rietveldtotensor: Program for processing powder X-Ray diffraction data under variable conditions. Glass Phys. Chem., 2018, 44 (1), P. 33-40.

[64] Berezhnaya M.V., Al'myasheva O.V., et al. Sol-gel synthesis and properties of $\mathrm{Y}_{1-x} \mathrm{Ba}_{x} \mathrm{FeO}_{3}$ nanocrystals. Russ. J. Gen. Chem., 2018, 88 (4), P. 626-631.

[65] Livage J., Sanchez C. Towards a soft and biomimetic nanochemistry. Actualite Chimique, 2005, 290-291, P. 72-76.

[66] Van-der-Vaal's I.D., Konstamm F. Lehrbuch der Thermodynamik. Maas and van Suchtelen. Leipzig, $1908,1,207$.

[67] Samsonov V.M., Murav'ev S.D., Bazulev A.N. Surface characteristics, structure and stability of nanosized particles. Russ. J. Phys. Chem. $A, 2000,74$ (11), P. 1791-1795.

[68] Magomedov M.N. Dependence of the surface energy on the size and shape of a nanocrystal. Physics of the Solid State, 2004, 46 (5), P. 954-968.

[69] Tolman R.C. The effect of droplet size on surface tension. J. Chem. Phys. A, 1949, 17 (203), P. $333-337$.

[70] Bykov T.V., Shchekin A.K. Surface tension, tolman length, and effective rigidity constant in the surface layer of a drop with a large radius of curvature. Inorg. Mater., 1999, 35 (6), P. 641-644.

[71] Krasnenko T.I., Rotermel M.V., Samigullina R.F. Stabilizing the associated non-autonomous phase upon thermal expansion of $\mathrm{Zn}_{2} \mathrm{~V}_{2} \mathrm{O}_{7}$. Russ. J. Inorg. Chem., 2017, 62 (4), P. 413-417.

[72] Tovbin Yu.K. Lower size boundary for the applicability of thermodynamics. Russ. J. Phys. Chem. A, 2012,86 (9), P. 1356-1369.

[73] Almjasheva O.V., Gusarov V.V. Metastable clusters and aggregative nucleation mechanism. Nanosystems: Phys. Chem. Math., 2014, 5 (3), P. 405-417.

[74] Neiman A.Ya., Uvarov N.F., Pestereva N.N. Solid state surface and interface spreading: An experimental study. Solid State Ionics, 2007, 177 (39-40), P. 3361-3369.

[75] Gusarov V.V. The thermal effect of melting in polycrystalline systems. Thermochim. Acta, 1995. 256 (2), P. $467-472$.

[76] Andrievski R.A. Review of thermal stability of nanomaterials. J. Mater. Sci., 2014, 49 (4), P. 1449-1460.

[77] Zhao M., Jiang Q. Melting and surface melting of low-dimensional in crystals. Solid State Communication, 2004,130, P. $37-39$.

[78] Gusarov V.V. Statics and dynamics of systems based on polycrystalline refractory oxides. Sc. D. Thesis, St. Petersburg State Technological Institute (Technical University), St. Petersburg, 1996, 44 p. (in Russian). 
[79] Gusarov V.V., Suvorov S.A. Transformations of nonautonomous phases and densification of polycrystalline systems. Journal of Applied Chemistry of the USSR, 1992, 65 (7), P. 1227-1235.

[80] Gusarov V.V. Kinetic features of heat effect of melting in polycrystalline systems. Russ. J. Appl. Chemistry, 1994, 67 (3), P. 364-366.

[81] Gusarov V.V., Suvorov S.A. Rapid thermal packing of materials. Russ. J. Appl. Chem., 1993, 66 (3), P. $431-437$

[82] Gusarov V.V., Suvorov S.A. Thickness of 2-dimentional nonautonomous phases in local equilibrium polycrystalline systems based on a single bulk phase. Russ. J. Appl. Chem., 1993, 66 (7), P. 1212-1216.

[83] Kikuchi R., Cahn J.W. Grain boundary melting transition in a two dimensional lattice gas model. Phys. Rev. B, 1980, 21 (5), P. 1893-1897.

[84] Gusarov V.V., Popov I.Yu. Flows in two-dimensional nonautonomous phases in polycrystalline systems. Nuovo Cim. D, 1996,18 (7), P. 799-805.

[85] Levitas V.I. Effect of the ratio of two nanosize parameters on the phase transformations. Scripta Materialia, 2018,192, P. $155-162$.

[86] Frenken J.W.M, van der Veen J.F. Observation of surface melting. Phys. Rev. Lett., 1985, 54, P. 134-141.

[87] Dash J.G. Surface melting. Contemp. Phys., 1989, 30 (2), P. 89-100.

[88] Zhou F., Lee J., Dallek S., Lavernia E.J. High grain size stability of nanocrystalline Al prepared by mechanical attrition. J. Mater. Res., 2001, 16, P. 3451-3458.

[89] Zhou F., Lee J., Lavernia E.J. Grain growth kinetics of a mechanically milled nanocrystalline Al. Scripta Materialia, 2001, 44 (8-9), P. 2013-2017.

[90] Rao C.N.R. Kinetics and thermodynamics of the crystal structure transformation of spectroscopically pure anatase to rutile. Can. J. Chem., 1961, 39, P. 498-500.

[91] Etters R.D., Kaelberer J. Character of melting transition in small atomic aggregates. J. of Chemical Physics, 1977,66 (11), P. 5112-5116.

[92] Zhou Z., Guo L., et al. Hydrothermal synthesis and magnetic properties of multiferroic rare-earth orthoferrites. Journal of Alloys and Compounds, 2014, 583, P. 21-31.

[93] Mendona E.C., Tenório M.A., et al. Intrinsic dependence of the magnetic properties of $\mathrm{CoFe}_{2} \mathrm{O}_{4}$ nanoparticles prepared via chemical methods with addition of chelating agents. J. Magn. Magn. Mater, 2015, 395, P. 345-349.

[94] Davar F., Hassankhani A., Loghman-Estarki M.R. Controllable synthesis of metastable tetragonal zirconia nanocrystals using citric acid assisted sol-gel method. Ceramics International, 2013, 39 (3), P. 2933-2941.

[95] Bachina A., Ivanov V.A., Popkov V.I. Peculiarities of $\mathrm{LaFeO}_{3}$ nanocrystals formation via glycine-nitrate combustion. Nanosystems: Phys. Chem. Math., 2017, 8 (5), P. 647-653.

[96] Phokha S., Pinitsoontorn S., Maensiri S., Rujirawat S. Structure, optical and magnetic properties of $\mathrm{LaFeO}_{3}$ nanoparticles prepared by polymerized complex method. J. Sol-Gel science and technology, 2014, 71 (2), P. 333-341.

[97] Proskurina O.V., Tomkovich M.V., et al. Formation of nanocrystalline $\mathrm{BiFeO}_{3}$ under hydrothermal conditions. Russ. J. Gen. Chem., 2017, 87 (11), P. 2507-2515.

[98] Dyachenko S.V., Martinson K.D., Cherepkova I.A. Particle size, morphology, and properties of transition metal ferrospinels of the MFe ${ }_{2} \mathrm{O}_{4}$ $(\mathrm{M}=\mathrm{Co}, \mathrm{Ni}, \mathrm{Zn})$ type, produced by glycine-nitrate combustion. Russ. J. Appl. Chem., 2016, 89 (4), P. $417-421$.

[99] Martinson K.D., Kondrashkova I.S., Popkov V.I. Synthesis of $\mathrm{EuFeO}_{3}$ nanocrystals by glycine-nitrate combustion method. Russ. J. Appl. Chem., 2017, 90 (8), P. 980-985.

[100] Kurdyukov D.A, Eurov D.A., et al. High-surface area spherical micro-mesoporous silica particles. Microporous Mesoporous Mater., 2016, 223, P. 225-229.

[101] Dideikin A.T., Aleksenskii A.E., et al. Rehybridization of carbon on facets of detonation diamond nanocrystals and forming hydrosols of individual particles. Carbon, 2017, 122, P. 737-745.

[102] Van-der-Vaal's I.D., Konstamm F. Course of Thermostatics. Moscow, ONTI, 1936, 452 p. (in Russian).

[103] Defay R., Prigogine I. Tension superficielle et adsorption. Desoer, Liege, 1951, 295 p.

[104] Defay R., Prigogine I., Sanfeld A. Surface thermodynamics. J. Colloid Interface Sci., 1977, 58 (3), P. $498-510$.

[105] Al'myasheva O.V., Gusarov V.V. Features of the phase formation in the nanocomposites. Russ. J. Gen. Chem., 2010,80 (3), P. 385-390.

[106] Al'myasheva O.V., Gusarov V.V. Nucleation in media in which nanoparticles of another phase are distributed. Dokl. Phys. Chem., 2009, 424 (2), P. 43-45.

[107] Popkov V.I., Almjasheva O.V., Gusarov V.V. The investigation of the structure control possibility of nanocrystalline yttrium orthoferrite in its synthesis from amorphous powders. Russ. J. Appl. Chem., 2014, 87 (10), P. 1417-1421.

[108] Bejan A. Review: J.D. Lewins, Thermodynamics: Frontiers and Foundations. $2009 . \quad$ URL: www. oecd-nea.org/dbprog/documents/Lewins_book_review.pdf.

[109] Hansen R.S. Surface and colloid science. Wiley, New York, 1969, 1, 260 p.

[110] Rusanov A.I. Phase equilibria and surface phenomena. Khimiya, Leningrad (1967). 388 p. (in Russian).

[111] Rusanov A.I. Thermodynamic fundamentals of mechanochemistry. Nauka, St. Petersburg, 2006, 221 p. (in Russian).

[112] Grinfeld M.A. Methods of continuum mechanics in theory of phase transformations. Nauka, Moscow, 1990, 312 p. (in Russian).

[113] Fletcher N.H. Surface structure of water and ice. Phil. Mag., 1962, 7, P. 255-259.

[114] Gilpin R.R. Wire regelation at low temperatures. Journal of Colloid and Interface Science, 1980, 77 (2), P. $435-448$.

[115] Clarke D.R. On the equilibrium thickness of intergranular glass phases in ceramic materials. J. Am. Ceram. Soc., 1987, 70, P. 15-22.

[116] Dash J.G. Thermomolecular pressure in surface melting: Motivation for frost heave. Science, 1989, 246, P. 1591-1593.

[117] Gusarov V.V., Suvorov S.A. Melting points of locally equilibrium surface phases in polycrystalline systems based on a single volume phase. J. Appl. Chem. of the USSR, 1990, 63 (8), P. 1560-1565.

[118] Gusarov V.V. Fast solid-phase chemical reactions. Zhurnal Obshchei Khimii, 1997, 67 (12), P. $1959-1964$.

[119] Almjasheva O.V. Heat-stimulated transformation of zirconium dioxide nanocrystals produced under hydrothermal conditions. Nanosystems: Phys. Chem. Math., 2015, 6 (5), P. 697-703.

[120] Vasilevskay A.K., Almjasheva O.V., Gusarov V.V. Peculiarities of structural transformations in zirconia nanocrystals. Journal of Nanoparticle Research, 2016, 18, P. 188-198.

[121] Almjasheva O.V. Formation and structural transformations of nanoparticles in the $\mathrm{TiO}_{2}-\mathrm{H}_{2} \mathrm{O}$ system. Nanosystems: Phys. Chem. Math., 2016, 7 (6), P. 1031-1049. 
[122] Murin I.V., Smirnov V.M., et al. Structural chemical transformations of $\alpha-\mathrm{Fe}_{2} \mathrm{O}_{3}$ upon transport reduction. Solid State Ionics, 2000, 133, P. 203-210.

[123] Zhang H., Banfield J. New kinetic model for the nanocrystalline anatase-to-rutile transformation revealing rate dependence on number of particles. American Mineralogist, 1999, 84 (4), P. 528-535.

[124] Bascom W.D., Cottington R.L., Singleterry C.R. Dynamic surface phenomena in the spontaneous spreading of oils on solids. American Chemical Society, Washington, DC, 1964, P. 355-379.

[125] Yannis K., Tassos B. Stability and dynamics of nonautonomous systems with pulsed nonlinearity. Phys. Rev. E, $2013, \mathbf{8 8}$ (4), 042924.

[126] Gilpin R.R. A model for the prediction of ice lensing and frost heave in soils. Water Resour. Res., 1980,16 (5), P. $918-930$.

[127] Nair R.R., Wu H.A., et al. Unimpeded permeation of water through helium-leak-tight graphene-based membranes. Science, 2012, 335, P. $442-444$.

[128] Murase Y., Kato E. Role of water vapour in crystallite growth and tetragonal - monoclinic phasetransformation of $\mathrm{ZrO}_{2}$. J. Am. Cream. Soc., 1983, 66 (3), P. 196-200.

[129] Wood G.R., Walton A.G. Homogeneous nucleation kinetics of ice from water. J. Appl. Phys., 1970,41 (7), P. $3027-3036$.

[130] Findenegg G.H., Jhnert S., Akcakayiran D., Schreiber A. Freezing and melting of water confined in silica nanopores. Chemphyschem., 2008., 9 (18), P. 2651-2659.

[131] Lee Penn R., Banfield J. Oriented attachment and growth, twinning, polytypism and formation of metastable phases; insights from nanocrystalline $\mathrm{TiO}_{2}$. American Mineralogist, 1998, 83 (9-10), P. 1077-1082.

[132] Grulke E.A., Yamamoto K., et al. Size and shape distributions of primary crystallites in titania aggregates. Advanced Powder Technology, 2017, 28 (7), P. 1647-1659.

[133] Tugova E.A., Karpov O.N. Nanocrystalline perovskite-like oxides formation in $\mathrm{Ln}_{2} \mathrm{O}_{3}-\mathrm{Fe}_{2} \mathrm{O}_{3}-\mathrm{H}_{2} \mathrm{O}$ ( $\mathrm{Ln}=\mathrm{La}$, Gd) systems. Nanosystems: Phys. Chem. Math., 2014, 5 (6), P. 854-860.

[134] Reiss H., Katz J.L., Cohen E.R. Translation-rotation paradox in the theory of nucleation. J. Chem. Phys., 1968, 48 (12), P. 5553-5560.

[135] Blinova I.V., Gusarov V.V., Popov I.Yu. A model of irregular impurity at the surface of nanoparticle and catalytic activity. Commun. Theor: Phis., 2012, 58 (1), P. 55-58.

[136] Tammann G. Über die abhngigkeit der zahl der kerne, welche sich in verschiedenen unterkhltenflssigkeiten bilden, von der temperature. Zeit. f. Physik. Chemie, 1898, 25, P. 441.

[137] Tammann G. Aggregatzustände. Verlag von Leopold Voss, Leipzig, 1922, 237 p.

[138] Gusarov V.V., Suvorov S.A. Autocatalytic solid-phase reaction of chrysoberyl formation. Zhurnal obshchei Khimii, 1988, 58 (4), P. 932934.

[139] Gusarov V.V., Suvorov S.A. Transformation and transport processes in polycrystalline systems and creep of materials. Journal of Applied Chemistry of the USSR, 1992, 65 (10), P. 1961-1964 (in Russian).

[140] Gusarov V.V., Malkov A.A., Malygin A.A., Suvorov S.A. Thermally activated transformations of 2D nonautonomous phases and contradiction of polycrystalline oxide materials. Inorganic Materials, 1995, 31 (3), P. 320-323.

[141] Zvereva I.A., Otrepina I.V., et al. Mechanism of formation of the complex oxide $\mathrm{Gd}_{2} \mathrm{SrFe}_{2} \mathrm{O}_{7}$. Russ. J. Gen. Chem., 2007, 77 (6), P. 973-978.

[142] Tugova E.A., Gusarov V.V. Peculiarities of layered perovskite-related $\mathrm{GdSrFeO}_{4}$ compound solid state synthesis. Journal of Alloys and Compounds, 2011, 509 (5), P. 1523-1528.

[143] Chupakhina T.I., Melkozerova M.A., Bazuev G.V. Phase formation features and magnetic properties of complex oxides in the systems Sr-Co-M-O (M = Zn, Cu). Russ. J. Inorg. Chem., 2013, 58 (3), P. 253-258.

[144] Tugova E.A. New DySrAlO 4 compound synthesis and formation process correlations for $\mathrm{LnSrAlO}_{4}(\mathrm{Ln}=\mathrm{Nd}$, Gd, Dy) series. Acta Metallurgica Sinica (English Letters),2016, 29(5), P. 450-456.

[145] Morozov M.I., Lomanova N.A., Gusarov V.V. Specific features of $\mathrm{BiFeO}_{3}$ formation in a mixture of bismuth (III) and iron (III) oxides. Russ. J. Gen. Chem., 2003, 73 (11), P. 1676-1680.

[146] Tugova E.A., Gusarov V.V. Processing stages of $\mathrm{Gd}_{2} \mathrm{Sr}_{(}\left(\mathrm{Al}_{1-x} \mathrm{Fe}_{x}\right)_{2} \mathrm{O}_{7}$ series. Rare Metals, 2014,33 (1), P. 47-53.

[147] Boldyrev V.V. Reactivity of solids. J. of Thermal Analysis, 199, 40, P. 1041- 1062.

[148] Baranchikov A.Ye., Ivanov V.K., Tretyakov Yu.D. Kinetics and mechanism of nickel ferrite formation under high temperature ultrasonic treatment. Ultrasonics Sonochemistry, 2007, 14 (2), P. 131-134.

[149] Tretyakov Yu.D. Self-organisation processes in the chemistry of materials. Russ. Chem. Rev., 2003 , 72 (8), P. 651-679.

[150] Kalinin S.V., Vertegel A.A., Oleynikov N.N., Tretyakov Y.D. Kinetics of solid state reactions with fractal reagent. J. Mater. Synth. Process, 1998, 6 (5), P. 305-309.

[151] Khvostova L.V., Volkova N.E., Gavrilova L.Ya, Cherepanov V.A. Crystal structure, oxygen nonstoichiometry and properties of novel Ruddlesden-Popper phase $\mathrm{Sm}_{1.8} \mathrm{Sr}_{1.2} \mathrm{Fe}_{2} \mathrm{O}_{7}-\delta$. Materials Letters, 2018, 213, P. 158-161.

[152] Yi L., Liu X.Q., Chen X.M. Crystal structure and infrared reflection spectra of $\mathrm{SrLn}_{2} \mathrm{Al}_{2} \mathrm{O}_{7}(\mathrm{Ln}=\mathrm{La}, \mathrm{Nd}, \mathrm{Sm}) \mathrm{microwave}$ dielectric ceramics. Int. J. Appl. Ceram. Technol., 2015, 12 (3), P. E33-E40.

[153] Chupakhina T.I., Mel'nikova N.V., Yakovleva E.A., Nikitina Yu.A. La ${ }_{1.8} \operatorname{Sr}_{0.2} \mathrm{Ni}_{0.8} \mathrm{M}_{0.2} \mathrm{O}_{4}(\mathrm{M}=\mathrm{Fe}$, Co, or Cu) complex oxides: synthesis, structural characterization, and dielectric properties. Russ. J. Inorg. Chem., 2018, 63 (2), P. 141-148.

[154] Fan X. Ch., Chen X. M., Liu X. Qi. Structural dependence of microwave dielectric properties of $\mathrm{SrRAlO}_{4}\left(\mathrm{R}=\mathrm{Sm}_{\mathrm{N}} \mathrm{Nd}\right.$, La) ceramics: crystal structure refinement and infrared reflectivity study. Chem. Mater, 2008, 20, P. 4092-4098.

[155] Tugova E.A. P/RS intergrowth type phases in the $\mathrm{Ln}_{2} \mathrm{O}_{3}-\mathrm{MO}-\mathrm{Al}_{2} \mathrm{O}_{3}$ systems. Russ. J. Gen. Chem., 2016,86 (11), P. $2410-2417$.

[156] Karita R., Kusaba H., Sasaki K., Teraoka Y. Superiority of nitrate decomposition method for synthesis of $\mathrm{K}_{2} \mathrm{NiF}_{4}-$ type $\mathrm{La}_{x} \mathrm{Sr}_{2}-{ }_{x} \mathrm{MnO}_{4}$ catalysts. Catalysis Today, 2007, 126, P. 471-475.

[157] Zhou J., Chen Y., Chen G., Wu K., Cheng Y. Evaluation of $\mathrm{La}_{x} \mathrm{Sr}_{2-x} \mathrm{FeO}_{4}$ layered perovskite as potential electrode materials for symmetrical solid oxide fuel cells. Journal of Alloys and Compounds, 2015, 647, P. 778-783.

[158] Liu Y.Y., Chen X.M., Liu X.Q. Magnetic properties and magnetoresistance of polycrystalline $\mathrm{SrLaCoO}_{4}$. Solid State Communications, 2005, 136, P. 576-579.

[159] Liu X.Q., Lü X.J., Chen X.M., Lü G.L. Structures and electrical conductivity of CaNdFeO 4 ceramics. J. Electroceram., 2008, 21, P. $487-490$. 
[160] Patel R., Simon Ch., Weller M.T. $\mathrm{LnSrScO}_{4}$ (Ln, La, Ce, Pr, Nd and Sm) systems and structure correlations for $\mathrm{A}_{2} \mathrm{BO}_{4}\left(\mathrm{~K}_{2} \mathrm{NiF}_{4}\right)$ structure types. J. Solid State Chem., 2007, 180, P. 349-359.

[161] Ganguli D. Cationic radius ratio and formation of $\mathrm{K}_{2} \mathrm{NiF}_{4}$-type compounds. J. Solid State Chem., $1979, \mathbf{3 0}$, P. $353-356$.

[162] Tugova E.A., Klyndyuk A.I., Gusarov V.V. Synthesis of solid solutions of double layered Ruddlesden-Popper phases in the Gd ${ }_{2} \mathrm{O}_{3}-\mathrm{SrO}_{-}$ $\mathrm{Fe}_{2} \mathrm{O}_{3}-\mathrm{Al}_{2} \mathrm{O}_{3}$ system. Russian Journal of Inorganic Chemistry, 2013, 58 (7), P. 848-854.

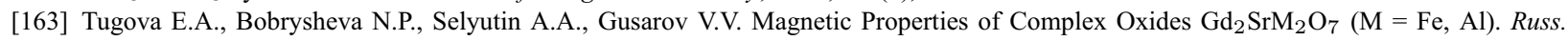
J. Gen. Chem., 2008, 78 (11), P. 2000-2001.

[164] Zvereva I., Smirnov Yu., et al. Complex aluminates $\mathrm{RE}_{2} \mathrm{SrAl}_{2} \mathrm{O}_{7}$ ( $\mathrm{RE}=\mathrm{La}, \mathrm{Nd}, \mathrm{Sm}-\mathrm{Ho}$ ): Cation ordering and stability of double perovskite slab-rocksalt layer $\mathrm{P}_{2} / \mathrm{RS}$ intergrowth. J. Solid State Sci., 2003, 5, P. 343-349.

[165] Chupakhina T.I., Melnikova N.V., Gyrdasova O.I. Synthesis, structural characteristics and dielectric properties of a new $\mathrm{K}_{2} \mathrm{NiF}_{4}$-type phase $\mathrm{Sr}_{2} \mathrm{Mn}_{0.5} \mathrm{Ti}_{0.5} \mathrm{O}_{4}$. Journal of Alloys and Compounds, 2016, 670, P. 105-112.

[166] Chupakhina T.I, Bazuev G.V. Synthesis and magnetic properties of $\mathrm{LaSr}_{2} \mathrm{CoMnO}_{7}$. Russ. J. Inorg. Chem., 2008 , 53 (5), P. 681-685.

[167] Aksenova T.V., Vakhromeeva A.E., et al. Phase equilibria, crystal structure, oxygen nonstoichiometry and thermal expansion of complex oxides in the $\mathrm{Nd}_{2} \mathrm{O}_{3}-\mathrm{SrO}-\mathrm{Fe}_{2} \mathrm{O}_{3}$ system. J. Solid State Chem., 2017, 251, P. 70-78.

[168] Feng J., Wan Ch., et al. Calculation of the thermal conductivity of $\mathrm{L}_{2} \mathrm{SrAl}_{2} \mathrm{O}_{7}$ (L = La, Nd, Sm, Eu, Gd, Dy). Phys. Rev. B, 2011, 84, 024302 .

[169] Tugova E.A. A comparative analysis of the processes of formation of Ruddlesden-Popper phases in the $\mathrm{La}_{2} \mathrm{O}_{3}-\mathrm{SrO}-\mathrm{M}{ }_{2} \mathrm{O}_{3}(\mathrm{M}=\mathrm{Al}, \mathrm{Fe})$ Systems. Glass Physics and Chemistry, 2009, 35 (4), P. 422-428.

[170] Zvereva I.A., Tugova E.A., et al. The impact of $\mathrm{Nd}^{3+} / \mathrm{La}^{3+}$ substitution on the cation distribution and phase diagram in the La $\mathrm{SrAl}_{2} \mathrm{O}_{7}-$ $\mathrm{Nd}_{2} \mathrm{SrAl}_{2} \mathrm{O}_{7}$ system. Chimica Techno Acta, 2018, 5 (1), P. 80-85.

[171] Petrosyan A.G., Popova V.F., et al. The $\mathrm{Lu}_{2} \mathrm{O}_{3}-\mathrm{Al}_{2} \mathrm{O}_{3}$ system: relations for equilibrium-phase and supercooled states. J. Cryst. Growth, 2006, 293, P. 74-77.

[172] Zvereva I.A., Pylkina N.S., et al. Phase equilibria in the $\mathrm{Gd}_{2} \mathrm{O}_{3}-\mathrm{SrAl}_{2} \mathrm{O}_{4}$ system. Glass Physics and Chemistry, 2005,31 (6), P. 808-811.

[173] Popova V.F., Tugova E.A., Gusarov V.V., Zvereva I.A. Phase equilibria in the $\mathrm{LaAlO}_{3}-\mathrm{LaSrAlO}_{4}$ system. Glass Physics and Chemistry, 2004, 30 (6), P. 564-567.

[174] Kovalenko A.N. High-temperature superconductivity: From macro- to nanoscale structures. Nanosystems: Phys. Chem. Math., 2016, 7 (6), P. 941-970

[175] Novoselov A.V., Drobot D.V. Synthesis of substrate materials based on rare-earth-containing mixed-oxide solid solutions for epitaxy of high-Tc superconductors. Inorganic Materials, 2009, 45 (6), P. 589-595.

[176] Zvereva I.A., Reznitskii L.A., Filippova S.E. Thermochemical and structural aspects of the stability of lamellar structures. Russ. J. Gen. Chem., 2004, 74 (5), P. 659-662.

[177] Pajaczkowska A., Novosselov A.V., Zimina G.V. On the dissociation and growth of $\mathrm{SrLaGaO}_{4}$ and $\mathrm{SrLaAlO}_{4}$ single crystals. Journal of Crystal Growth, 2001, 223, P. 169-174.

[178] Smirnov Yu.E., Zvereva I.A. Cation distribution and interatomic interactions in oxides with heterovalent isomorphism: III.1 Complex aluminates $\mathrm{LnCaAlO}_{4}(\mathrm{Ln}=\mathrm{Y}, \mathrm{La}, \mathrm{Nd}, \mathrm{Gd}, \mathrm{Ho}$, Er, Yb). Russ. J. Gen. Chem., 2001, 71 (6), P. 845-852.

[179] Chen X.M., Xiao Y., Liu X.Q., Hu X. $\mathrm{SrLnAlO}_{4}$ (Ln Nd and Sm) microwave dielectric ceramics. Journal of Electroceramics, 2003, 10, P. 111-115.

[180] Rao C.N.R., Raveau B. Transition Metal Oxides: Structure, Properties and Synthesis of Ceramic Oxides. Wiley-VCH, New York, 1998, $227 \mathrm{p}$.

[181] Meskin P.E., Ivanov V.K., et al. Ultrasonically assisted hydrothermal synthesis of nanocrystalline $\mathrm{ZrO}_{2}, \mathrm{TiO}_{2}, \mathrm{NiFe}_{2} \mathrm{O}_{4}$ and $\mathrm{Ni}_{0.5} \mathrm{Zn}_{0.5} \mathrm{Fe}_{2} \mathrm{O}_{4}$ powders. Ultrasonics Sonochemistry, 2006, 13 (1), P. 47-53.

[182] Bugrov A.N., Almjasheva O.V. Effect of hydrothermal synthesis conditions on the morphology of $\mathrm{ZrO}_{2}$ nanoparticles. Nanosystems: Phys. Chem. Math., 2013, 4, P. 810-815.

[183] Li Y., Xu S. Hydrothermal synthesis of lanthanide hydroxide micro/nanorods in presence of tetrabutylammonium hydroxide. Journal of Rare Earths, 2016, 34 (6), P. 618-625.

[184] Gusev A.I., Kurlov A.S. Production of nanocrystalline powders by high-energy ball milling: model and experiment. Nanotechnology, 2008, 19 (26), P. 265302-265310.

[185] Komarneni S., Tsuji M., Wada Y., Tamaura Y. Nanophase ferrites for $\mathrm{CO}_{2}$ greenhouse gas decomposition. J. Mater. Chem., 1997, 7 (12), P. 2339-2340.

[186] Tretyakov Y.D., Oleinikov N.N., et al. Self-organization in physicochemical systems - on the path to creating novel materials. Inorg. Mater, 1990, 30 (3), P. 277-290.

[187] Pomogailo A.D., Rozenberg A.S., Dzhardimalieva G.I. Thermolysis of metallopolymers and their precursors as a method for the preparation of nanocomposites. Russ. Chem. Rev., 2011, 80 (3), P. 257-292.

[188] Vanetsev A.S., Tretyakov Yu.D. Microwave-assisted synthesis of individual and multicomponent oxides. Russ. Chem. Rev., 2007, 76 (5), P. 397-413.

[189] Baranchikov A.Ye., Ivanov V.K., Tretyakov Yu.D. Sonochemical synthesis of inorganic materials. Russ. Chem. Rev., 2007, 76 (2), P. 133-151.

[190] Lomanova N.A., Tomkovich M.V., Sokolov V.V., Gusarov V.V. Special features of formation of nanocrystalline BiFeO 3 via the glycinenitrate combustion method. Russ. J. Gen. Chem., 2016, 86 (10), P. 2256-2262.

[191] Türk M. Particle formation with supercritical fluids: challenges and limitations (Chapter 7). Elsevier, $2014,152 \mathrm{p}$.

[192] Tretyakov Yu.D., Lukashin A.V. Eliseev A.A. Synthesis of functional nanocomposites based on solid-phase nanoreactors. Russ. Chem. Rev., 2004, 73 (9), P. 899-921.

[193] Krasilin A.A., Gusarov V.V. Redistribution of $\mathrm{Mg}$ and $\mathrm{Ni}$ cations in crystal lattice of conical nanotube with chrysotile structure. Nanosystems: Phys. Chem. Math., 2017, 8, P. 620-627.

[194] Korytkova E.N., Semyashkina M.P, et al. Synthesis and growth of nanotubes $\mathrm{Mg}_{3} \mathrm{Si}_{2} \mathrm{O}_{5}(\mathrm{OH}, \mathrm{F})_{4}$ composition under hydrothermal conditions. Glass Phys. Chem., 2013, 39 (3), P. 294-300. 
[195] Gulina L.B., Tolstoy V.P., Kasatkin I.A., Murin I.V. Facile synthesis of scandium fluoride oriented single-crystalline rods and urchin-like structures by a gas-solution interface technique. Crystengcomm., 2017, 19 (36), P. 5412-5416.

[196] Komarneni S. Nanophase materials by hydrothermal, microwave-hydrothermal and microwave solvothermal methods. Current Science, 2003, 85 (12), P. 1730-1734.

[197] Brinker C.J., Scherer G.W. Sol-Gel Science: The Physics and Chemistry of Sol-Gel Processing. Academic Press, San Diego, 1990, 908 p.

[198] Tugova E., Yastrebov S., Karpov O., Smith R. $\mathrm{NdFeO}_{3}$ nanocrystals under glycine nitrate combustion formation. Journal of Crystal Growth, 2017, 467, P. 88-92.

[199] Komlev A.A., Vilegzhaninov E.F., et al. Formation of spinel particles in the Mg-Al-O-N system in the combustion wave front. Bulletin of the Saint Petersburg State Institute of Technology (Technical University), 2013, 1, P. 3-6.

[200] Ivleva T.P., Merganov A.G. Three-dimensional spinning waves of gasless combustion. Doklady Akademii Nauk, 2000, 371 (6), P. 753758.

[201] Bhavani P., Rajababu C.H., et al. Synthesis of high saturation magnetic iron oxide nanomaterials via low temperature hydrothermal method. Journal of Magnetism and Magnetic Materials, 2017, 426, P. 459-466.

[202] Adachi M., Okuyama K., Seinfeld J.H. Experimental studies of ion-induced nucleation. J. Aerosol. Sci., 1992,23 (4), P. $327-337$.

[203] Fedoseev V.B., Fedoseeva E.N. Size effects during phase transformations in stratifying systems. Russ. J. Phys. Chem. A, 2014, 88 (3), P. 436-441.

[204] Oxtoby D.W. Nucleation of first-order phase transitions. Acc. Chem. Res., 1998, 31 (2), P. 91-97.

[205] Ilhan S., Izotova S.G., Komlev A.A. Synthesis and characterization of $\mathrm{MgFe}_{2} \mathrm{O}_{4}$ nanoparticles prepared by hydrothermal decomposition of co-preciptated magnesium and iron hydroxides. Ceramics International, 2015, 41 (1), P. 577-585.

[206] Gandhi A.S., Jayaram V. Plastically deforming amorphous $\mathrm{ZrO}_{2}-\mathrm{Al}_{2} \mathrm{O}_{3}$. Acta materialia, 2003, 51, P. $1641-1649$.

[207] Ding X.-Z., Liu X.-H. Correlation between anatase-to-rutile transformation and grain growth in nanocrystalline titania powders. J. Mater. Res., 1998, 13 (9), P. 2556-2559.

[208] Lawrencaen P.J., Parker S.C. Computer simulation studies of perfect and defective surfaces in Cr $2 \mathrm{O}_{3} . J$. Am. Ceram. Soc., 1988, 71 (8), P. 389-391.

[209] Rebinder P.A., Shchukin E.D. Surface phenomena in solids during the course of their deformation and failure. Sov. Phys. Usp., 1973, 15 (5), P. 533-554.

[210] Oxtoby D.W. Phase transitions: Catching crystals at birth. Nature, 2000, 406, P. 464-465.

[211] Rotermel M.V., Krasnenko T.I., Mechanism of thermal expansion of structural modifications of zinc pyrovanadate. Crystallography Reports, 2017, 62 (5), P. 703-709.

[212] Tauson V.L., Loginov B.A., Akimov V.V., Lipko S.V. Nonautonomous phases as potential sources of incompatible elements. Doklady Earth Sciences, 2006, 407 (2), P. 280-283.

[213] Sdobnyakov N.Yu., Samsonov V.M. On the size dependence of surface tension in the temperature range from melting point to critical point. CEJP, 2005, 3 (2), P. 247-250.

[214] Samsonov V.M., Dronnikov V.V., Mal'kov O.A. The size dependence of the melting temperature of nanocrystals. Russ. J. Phys. Chem., 2004, 78 (7), P. 1044-1047

[215] Tauson V.L., Akimov V.V. Introduction to the theory of forced equilibria: general principles, basic concepts, and definitions. Geochimica et Cosmochimica Acta, 1997, 61 (23), P. 4935-4943.

[216] Summ B.D., Samsonov V.M. Concepts of Rehbinder's school and modern theories of spreading. Colloids and surfaces a-physicochemical and engineering aspects, 1999, 160 (2), P. 63-77.

[217] Kondepudi D., Prigogine I. Modern Thermodynamics. From heat engines to dissipative structures. Wiley, UK, 1998,506 p.

[218] Kovalenko A. Inner nonstationarness of energy conversion in non-equilibrium thermodynamic system. Proceedings of the Fifth Baltic Heat Transfer Conference, 21-23 September 2009, St-Petersburg, SPbGPU, 2009, P. 446-453.

[219] Green S., Sengers J.V. Critical Phenomena. Proc. Conference on phenomena in the neighborhood of critical points, NBS Misc. Publ. 273, Washington, 1966, P. 1-242.

[220] Kadanoff L.P. Phase Transition and Critical Phenomena. Green. Acad. Press, New York, 1976, 5, P. 2-34

[221] Widom B. Equation of state in neighborhood of the critical point. J. Chem. Phys., 1965, 43 (11), P. $255-262$.

[222] Sedoi V.S., Valevich V.V. Production of highly dispersed metal powders by electrical explosion in reduced-pressure nitrogen. Technical Physics Letters, 1999, 25 (7), P. 584-585.

[223] Kovalenko A.N., Kalinin N.V. Thermodynamic instability of compound and formation of nanosized particles nearby the critical point of phase generating media. Nanosystems: Phys. Chem. Math., 2014, 5 (2), P. 258-293.

[224] Dulnev G.N., Novikov V.V. Processes of transfer in non-uniform environments. Energoatomizdat, Leningrad, 1991,248 p. (in Russian).

[225] Menshikov M.V., Molchanov S.A., Sidorenko A.F. Theory of a perkolyation and some applications. VINITI, Moscow, 1986, 24, P. 53-110 (in Russian).

[226] Burtsev V.A., Kalinin N.V. About conductivity at a stage actually explosion of conductors. Physics of extreme states. Works of the international conference "Interaction of intensive streams of energy with substance". Chernogoloka: RAS, KBGU, 2005, P. 156-158 (in Russian)

[227] Pervov V.S. Supramolecular ensembles in eutectic alloys. Russian Chemical Reviews, 2003, 72 (9), P. $759-768$.

[228] Pervov V.S., Mikheikin I.D., Shatilo Ya.V., Makhonina E.V. Supramolecular model of eutectics: Metastable states and structure of inorganic alloys. Russ. J. Inorg. Chem., 2007, 52 (4), P. 524-531.

[229] Len G.-M. Supramolecular chemistry. Concept and prospects. Science, Novosibirsk, 1998, 334 p.

[230] Pervov V.S., Manokhina E.V., Dobrokhotova Zh.V., Zotova A.E. Metastable states in inorganic systems. Inorg. Mater., 2011 , 47 (13), P. $1407-1427$

[231] Pervov V.S., Makhonina E.V., et al. Supramolecular model of eutectics: Functional materials based on nonautonomous phases. Inorg. Mater, 2009, 45 (12), P. 1382-1387.

[232] Frenkel Ya.I., Kontorova T.A. To the theory of plastic deformation and a dvoynikovaniye. ZhETF, 1938, 8 (12), P. $1340-1348$.

[233] Zavragnov A.Y., Makhonina E.V., Mikheykin I.D., Pervov V.S. Supramolecular model of eutectics. Metastable structures in inorganic systems and a possibility of their functionalization. Bulletin of the RFBR, 2011, 4 (72), P. $48-54$ (in Russian). 
[234] Lyapunova E.A., Uvarov S.V., et al. Modification of the mechanical properties of zirconium dioxide ceramics by means of multiwalled carbon nanotubes. Nanosystems: Phys. Chem. Math., 2016, 7 (1), P. 198-203.

[235] Saha S., Dutta A., Mukhopadhya P.K., Sinha T.P. Dielectric relaxation and charge transport process in $\mathrm{PrCrO}_{3}$ nano-ceramic. Nanosystems: Phys. Chem. Math., 2016, 7 (4), P. 613-617.

[236] Mezentseva L.P., Osipov A.V., et al. Chemical and thermal stability of phosphate ceramic matrices. Glass Phys. Chem., 2017, 43 (1), P. 83-90.

[237] Bechta S.V., Krushinov E.V., et al. Phase diagram of the $\mathrm{ZrO}_{2}-\mathrm{FeO}$ system. Journal of Nuclear Materials, 2006,348 (1-2), P. 114-121.

[238] Kazin P.E., Tretyakov Yu.D. Microcomposites based on superconducting cuprates. Russ. Chem. Rev., 2003,72 (10), P. 849-865.

[239] Kenges K.M., Proskurina O.V., et al. Synthesis and properties of nanocrystalline materials based on LaPO 4 . Russ. J. Appl. Chem., 2017, 90 (7), P. 1047-1054.

[240] Almjasheva O.V., Gusarov V.V., Kovalenko A.N., Ugolkov V.L. Nanocrystals of $\mathrm{ZrO}_{2}$ as sorption heat accumulators. Glass Phys. Chem., 2007, 33 (6), P. 587-589.

[241] Matsukevich I.V., Klyndyuk A.I., et al. Thermoelectric properties of $\mathrm{Ca}_{3-x} \mathrm{Bi}_{x} \mathrm{Co}_{4} \mathrm{O}_{9}+\delta(0.0 \leq x \leq 1.5)$ ceramics. Inorg. Mater., 2016, 52 (6), P. 593-599.

[242] Tretyakov Yu.D. Development of inorganic chemistry as a fundamental base for the design of new generations of functional materials. Russ. Chem. Rev., 2004, 73 (9), P. 831-846

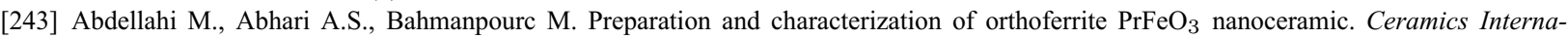
tional, 2016, 42 (4), P. 4637-4641.

[244] Tretyakov Yu.D., Goodilin E.A. Chemical principles of preparation of metall-oxide superconductors. Russ. Chem. Rev., 2000, 69 (1), P. 1-34.

[245] Pervov V.S., Ovchinnikova S.I., et al. Nanoionics: Principles of ceramic materials fabrication for electrochemical power generation. Inorg. Mater., 2016, 52 (1), P. 83-88.

[246] Andrievski R.A. The role of nanoscale effects in the interaction between nanostructured materials and environments. Protection of Metals and Physical Chemistry of Surfaces, 2013, 49 (5), P. 528-540.

[247] Rusanov A.I. Surface thermodynamics revisited. Surface Science Reports, 2005, 58, P. 111-239.

[248] Adamson A.W., Gast A.P. Physical chemistry of surfaces. 6th ed. A Wiley-Interscience Publication, N.Y., 1997,784 p.

[249] Prigogine I., Defay R. Chemical Thermodynamics. Longmans, Green and Co., New York, 1954, 409 p.

[250] Feder J. Fractals. Physics of Solids and Liquids. Plenum Press, New York, 1988, 305 p.

[251] Tauson V.L., Kravtsova R.G., Grebenshchikova V.I., Lustenberg E.E. Surface typochemistry of hydrothermal pyrite: Electron spectroscopic and scanning probe microscopic data. II. Natural pyrite. Geochemistry International, 2009 , 47 (3), P. $231-243$.

[252] Tauson V.L, Lipko S.V., Shchegolkov Yu.V. Surface nanoscale relief of mineral crystals and its relation to nonautonomous phase formation. Crystallography Reports, 2009, 54 (7), P. 1219-1227.

[253] Roldughin V.I. Self-assembly of nanoparticles at interfaces. Russ. Chem. Rev., 2004, 73 (2), P. 115-145.

[254] Kuzmenko A.P., Chakov V.V., Aung Ch.N. Operated self-assembly micro and nanostructures. Nanotechnics, 2013, 4 (36), P. 30-31 (in Russian)

[255] Paradisi P., Cesari R., Mainardi F., Tampieri F. The fractional Fick's law for non-local transport processes. Physica A, 2001, 293, P. 130-142.

[256] Vlad M.O. Fractional diffusion on fractals: Self-similar stationare solutions in a force field derived from a logarithmic potential. Chaos, Solutions \& Fractals, 1994, 4 (2), P. 191-199.

[257] Taukenova F.I., Shkhanukov-Lafishev M.Kh. Difference methods for solving boundary value problems for fractional differential equations. Computational Mathematics and Mathematical Physics, 2006, 46 (10), P. 1785-1795.

[258] Pleskachevsky Yu.M., Chigareva Yu.A. Distribution of temperature in a layer with fractal structure, 2015, URL: https ://journals.bntu.by/handle/data/14552.

[259] Uchaykin V.V. Automodel abnormal diffusion and steady laws. Phys. Usp., 2003, 46 (8) P. 821-849.

[260] Meylanov R.P., Shabanova M.R. The thermoconductivity equation for areas with fractal structure. Modern high technologies, 2007, 8, 6 (in Russian).

[261] Rényi A. On a new axiomatic theory of probability. Acta Mathematica Hungaria, 1955, 6, P. $285-335$.

[262] Klimontovich Yu.L. Entropy and information of open systems. Progress of physical sciences, 1999 , 169 (4), P. 443-452 (in Russian).

[263] Dozhdikova O.L., Zarichnyak Yu.P., et al. Anomalies of concentration dependence of heat conductivity of the baked compositions with an ultradisperse component in the TiN-AlN system. Powder metallurgy, 1992, 5 (in Russian).

[264] Dulnev G.N. Processes of transfer in non-uniform environments. ITMO University, Leningrad, 1985, 27 p. (in Russian).

[265] Dulnev G.N., Zarichnyak Yu.P. Heat conductivity of mixes and composite materials. Reference book. Energy, Leningrad, 1974,264 p. (in Russian).

[266] Dykhne A.M., Snarskii A.A., Zhenirovskii M.I. Stability and chaos in randomly inhomogeneous two-dimensional media and LC circuits. Phys. Uspekhi, 2004, 47 (8), P. 821-828 (in Russian).

[267] Galkin A.A., Lunin V.V. Water in sub- and supercritical states as a universal medium for chemical reactions. Russ. Chem. Rev., 2005, 74 (1), P. 21-35.

[268] Valyashko V.M. Equilibria with the participation of supercritical fluids. Supercritical Fluids. Theory and practices, 2006,1 , P. 10-26.

[269] Aymonier C., Loppinet-Serani A., et al. Review of supercritical fluids in inorganic materials science. Journal of Supercritical Fluids, 2006, 38, P. 242-251.

[270] Aimable A., Muhr H., et al. Continuous hydrothermal synthesis ofinorganic nanopowders in supercritical water: Towards a better control of the process. Powder Technology, 2009, 190, P. 99-106.

[271] Rekhviashvili S.Sh., Kishtikova E.V. On the size dependence of the surface tension. Technical Physics, 2011,56 (1), P. $143-146$.

[272] Abraham F.F. Re-examination of homogeneous nucleation theory: statistical thermodynamics aspects. J. Chem. Phys., 1968, 48 (2), P. 732-740.

[273] Lin J. Equilibrium distribution of droplets in the theory of nucleation. J. Chem. Phys., 1968, 48 (9), P. $4128-4130$. 
[274] Stillinger F.H. Comment on the translation-rotation paradox in the theory of irreversible condensation. J. Chem. Phys., 1968, 48 (3), P. $1430-1431$.

[275] Kuni F.M., Rusanov A.I. The homogeneous nucleation theory and the fluctuation of the center of mass of a drop. Phys. Letters A, 1969, 29 (6), P. 337-338.

[276] Reiss H. Treatment of drop like clusters by means of the classical phase integral in nucleation theory. J. Stat. Phys., 1970, 2 (1), P. 83-104.

[277] Blander M., Katz J.L. The thermodynamics of cluster formation in nucleation theory. J. Stat. Phys., 1972,4 (1), P. $55-59$.

[278] Girshick S.L. Comment on: Self-consistency correction to homogeneous nucleation theory. J. Chem. Phys., 1991, 94 (1), P. $826-828$.

[279] Katz J.L. Nucleation theory without Maxwell Demons. J. Coll. Interface Sci., 1977, 61 (2), P. 351-355.

[280] Garnier J.P., Mirabel P., Rabeony H. Experimental results of homogeneous nucleation of super saturated vapors. J. Chem. Phys., 1983, 79 (4), P. 2097-2098.

[281] Ruth V., Hirth J.P., Pound G.M. On the theory of homogeneous nucleation and spinodal decomposition in condensation from the vapor phase. J. Chem. Phys., 1988, 88 (11). P. 7079-7087.

[282] Auer S., Frencel D. Numerical predication of absolute crystallization rates in hard-sphere colloids. J. Chem. Phys., 2004, 120 (6), P. 3015-3029.

[283] Girshick S.L., Chiu C.P. Kinetic nucleation theory: A new expression for the rate of homogeneousnucleation from an ideal supersaturated vapor. J. Chem. Phys., 1990, 93 (2), P. 1273-1278.

[284] Zeng X.C., Oxtoby D.W. Gas-liquid nucleation in Lennard-Jones fluids. J. Chem. Phys., 1991,94 (6), P. $4472-4478$.

[285] Cahn J.W., Hilliard J.E. Free energy of a nonuniform system. III. Nucleation in a two-component incompressible fluid. J. Chem. Phys., 1959, 31 (3), P. 688-700.

[286] Lothe J., Pound G.M. Reconsideration of nucleation theory. J. Chem. Phys., 1962, 36 (8), P. $2080-2085$.

[287] Lothe J. Concentration of clusters in nucleation and the classical phase integral. J. Chem. Phys., 1968,48 (4), P. 1849-1852.

[288] Frenkel J.A. General theory of heterophase fluctuations and pretransition phenomena. J. Chem. Phys., 1939,7 (7), P. $538-546$.

[289] Volmer M. Kinetik der Phasenbildung. Angewandte Chemie, 1939, 30, P. 503-504.

[290] Tribus M. Thermostatics and thermodynamics. Energia, Moscow, 1970, 504 p. (in Russian). 